H5226$525 \%$

D5798 5813 . 
$5-507$ 
Wre ig; s.798

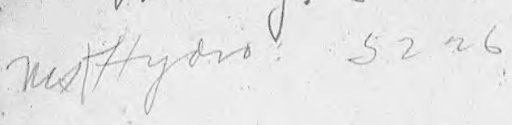


5796

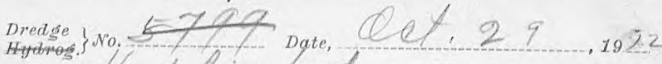

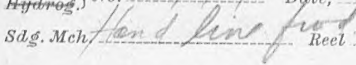

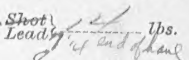

Turns Corn Depth fins.

Sd g. Cup Amt. Bot'm Character of
Bottom

Thermom. $\left\{\begin{array}{l}\text { Make } \\ \text { No. }\end{array}\right.$

Corn $\left\{\begin{array}{l}\text { Instr. } \\ \text { Stem. }\end{array}\right.$

Bottom Reading

Temp. Surf. $\left\{\begin{array}{l}5 \\ -1 \cdots\end{array}\right.$

Cord Temp.

Density: Surf.

Wind: Direction hus Force 0 Baronet. 30,24 Amt of 3 State of S

Weather

Time

Time
$\operatorname{Air}\left\{\begin{array}{l}58^{\circ} \\ \end{array}\right.$

fins.

Direction and Force
of Current. $\left\{\begin{array}{l}\text { Surface } \\ \text { Bottom }\end{array}\right.$

Tide

General Locality

Position

Chart used: No. $E d^{\prime} n$

Drift: Direction Distance

Depth of Haul Ballon

Apparatus and Rig used S'T hame wo th woods

rumen's glass loots. 1 mudbog

Time towing

n. 0 m.

Remarks:

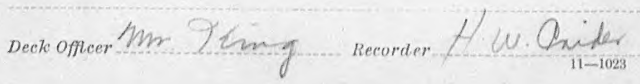


$11-1023$

SOUNDING WIRE.

M. Down. UP. .... M.

FMS DREDGING CABLE.

$P$ M. Down. UP. C. M.

$\begin{array}{ll}01257 & 00 / 2000\end{array}$

$35+00 / 25830 / / 838$

200

300

400

500

600

700

800

900

1000

1100

1200

1800

1400

1500

1600

1700

1800

1900

2000

2100

2200

2300

2400

2500

2600

2700

2800

2900

3000

3100

3200

3300

3400

3500

3600

3700

3800

3900

4000

4100

4200

4300

4400

4500

4600

4700

4800

4900 


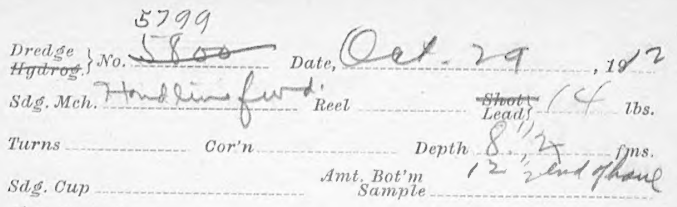

Character of

Bottom

Thermom. $\left\{\begin{array}{l}\text { Make } \\ \text { No. }\end{array}\right.$ Corn $\left\{\begin{array}{l}\text { Instr. } \\ \text { Stem. }\end{array}\right.$

Bottom Reading Cord Temp.

Temp. Surf. $\left\{\begin{array}{l}57^{0} \\ \end{array}\right.$

$\left.\begin{array}{l}\text { Time } 2,500\} \\ \text { Time }\end{array}\right\} \operatorname{sir}\left\{9^{\circ}\right.$

Density: Surf. fms.

Wind: Direction ...n:

Fores $0-1$

Baronet. 30,22 Ant of 3 State of SS S

Weather $\mathrm{bec}^{\mathrm{c}}$

Direction and Force
of Current. $\left\{\begin{array}{l}\text { Surface } \\ \text { Bottom }\end{array}\right.$

Tide

General Locality

Position

Chart used: No. Edh

Drift: Direction Distance

Depth of Haul $Q$ alton

Apparatus and Risused I' J one well

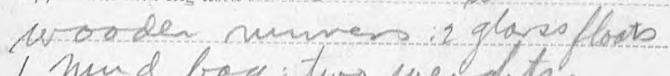

I mind bag: two weights.

Time towing

n. 20

$m$.

Remarks:

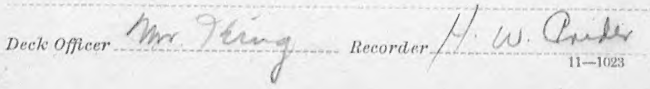


SOUNDING WIRE.

$\ldots$ M. Down, UP. _... M.
FMS.

DREDGING CABLE.

L. Down. Up. M.

$70100 / 583021830$

200

300

400

500

600

700

800

900

1000

1100

1200

1300

1400

1500

1600

1700

1800

1900

2000

2100

2200

2300

2400

2500

2600

2700

2800

2900

3000

8100

3200

3300

3400

3500

8600

3700

3800

3900

4000

4100

4200

4800

4400

4500

4600

4700

4800

4900 
5800

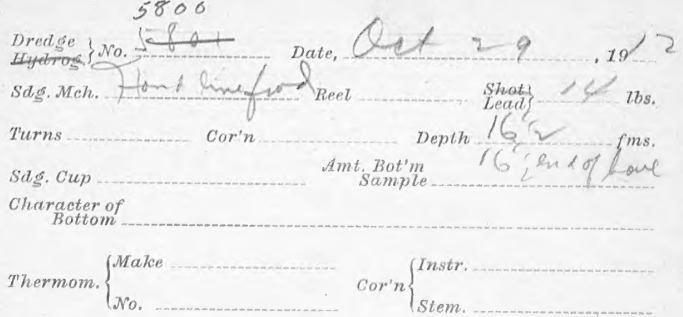

Bottom Reading

Cgr'd Temp.

Temp. Surf. \{

Time 3:07

$$
\operatorname{sir}\left\{9^{\circ}\right.
$$

Density: Surf.

fins.

Wind: Direction ...S $\omega$

Force 0 .

Baromet. $35: 22$ Action 3 State of 3

Weather 0

Direction and Force
of Current. $\left\{\begin{array}{l}\text { Surface } \\ \text { Bottom }\end{array}\right.$

Tide

General Locality

Position

Chart used: No. Eden

Drift: Direction Distance

Depth of Haul Patton

Apparatus and Rig used Quite due gl ¿ gar

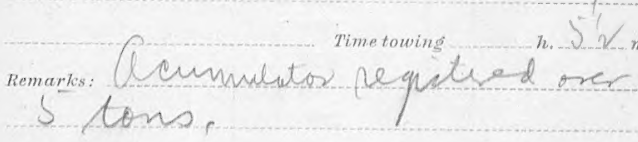

Deckoffeer Whathwaly Recorder 
SOUNDING WIRE.

M. Down. UP. M.
FMS. DREDGING CABLE.

r.

O. M. Down. Up. L M.

100

\begin{tabular}{l|l|l|l|l|l|}
3 & 02 & 00 & 3 & 17 & $45^{2}$ \\
3 & 05 & 50 & 13 & 15
\end{tabular}

3003063031200

300

400

500

600

700

800

900

1000

1100

1200

1300

1400

1500

1600

1700

1800

1900

2000

2100

2200

2300

2400

2500

2600

2700

2800

2900

3000

3100

3200

3300

3400

8500

3600

3700

3800

3900

4000

4100

4200

4300

4400

4500

4600

4700

4800

4900 
5801

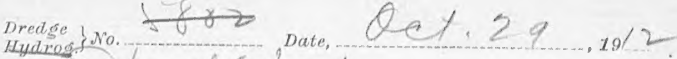

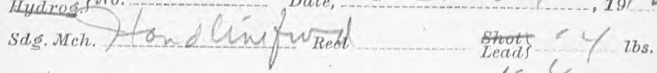

Turns Corn $\operatorname{Depth} / 6$ '

Sd s. Cup Ant. Bot'm $>7$ end f haul Character of
Bottom

Thermos. $\left\{\begin{array}{l}\text { Max } \\ \text { No. }\end{array}\right.$

$$
\text { Corn }\left\{\begin{array}{l}
\text { Instr. } \\
\text { Stem. }
\end{array}\right.
$$

Bottom Reading Cor'd Temp.

Temp. Surf. \{ Time Time $\operatorname{Air}\{$

Density: Surf. fms.

Wind: Direction ..S.U.

Force $x-1$ Baronet. 30.27 Amt. of $L I$ State of $S$ Weather

Direction and Force
of Current. $\left\{\begin{array}{l}\text { Surface } \\ \text { Bottom }\end{array}\right.$

Tide

General Locality

Position

Chart used: No. Eden

Drift: Direction Distance

Depth of Haul Bottom

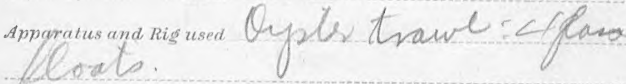
floats.

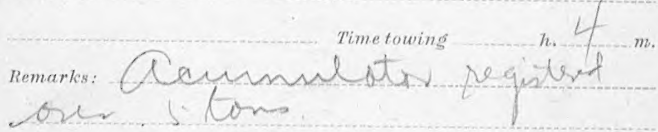

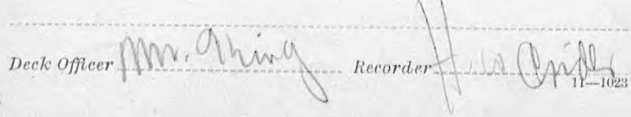


SOLNIDING WIIRE,

MT. Dowx. U1. II.
FMS.

DREDGING ('AIBLE

P.M. Dows. Vin. II.

1) $\because 93034230$

100

:30)

.100

5,00

(ion)

roc

8क्ष)

()

1000

1100

1200

1300

1.100

1500

(6)

1700

1800

19)(

$: 2(001)$

2100

2.201

$: 2: 200$

$\because 100$

ivio

2000

$2 \% 00$

2500

2900

3000

3100

$: 3900$

:3:30)

:) 100

$\sin (0)$

3600

:

8800

:3900

$.1000)$

.1100

(19)( $(1)$

4:190

4.100

45000

. $16,(10)$

1700

4500

(1919) 


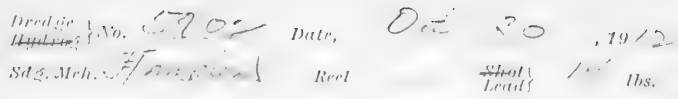
Turus
Cur'n
linplth
fins.

Sits. (in)

Ant. Bot'm

Character of
Pottom

Thermom. $\left\{\begin{array}{l}\text { Huk\% } \\ \text { No. }\end{array}\right.$

Cor'n $\left\{\begin{array}{l}\text { lnstr. } \\ \text { Stem. }\end{array}\right.$

Bottom Reading Cor'd Temp.
Temp. Surf.
rimr
Time
$\{\operatorname{Air}\}$

7)ensity: Surf.

Wina: Direction.

firromer.? $0, \geq 8$

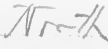

fins.

Wrethen

Amt of

$0-1$

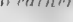

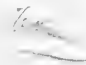

State of

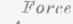

Direction and Force
of Current. $\left\{\begin{array}{l}\text { Surface } \\ \text { Jiottom }\end{array}\right.$

Tide

General Locality

Posifiul

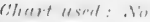

$\left.E I^{\prime}\right)$

Drift: Direction

Disiance

liopith of llanl

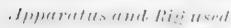

Timetruthet

h.
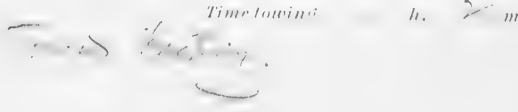

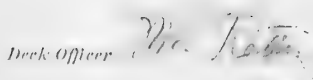


SOUNDING WVIIRT:

ML. D()WN. UI? M.

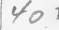




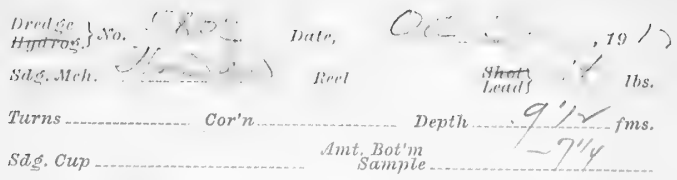

$$
\begin{aligned}
& \begin{array}{c}
\text { Character of } \\
\text { Dollom }
\end{array}
\end{aligned}
$$

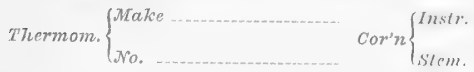

\section{(ior'd. Trmp)}

$$
\begin{aligned}
& \left\{A i v^{*}\right\}
\end{aligned}
$$

fins.

Densily: surf.

Wind: Direction

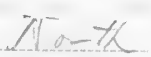

Baromet. 30.27

Amt. of

Force 0 ,

Weather

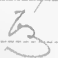

State of Sea.

\section{Direction and Force
of Current. $\left\{\begin{array}{l}\text { Surform } \\ \text { lioltom }\end{array}\right.$}

Title.

\section{General Lowelity}

\section{- Position}

linart uset: . Ho

Eitin

Drift: Direction Distance

Depth of Haul.

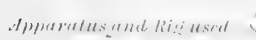

2

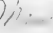


$11-10: 3$

\section{SOUNDING WIRE.}

.... M, Down, UP. _ . M.

L, 44

2010

$: 3()()$

(10)

iv()

(i) () ()

รi) ()

$\mathrm{xil}(\mathrm{)}$

(t)()()

1000

110()

$120(1)$

1:30)

1.100

1500

1000

1700

1800

1900

$20(0)$

2100

2000

2:300

2100

280

(2)(it)

$27(1)$

ख्र(1)0

ㅁำ

$: 3000$

$\$ 100)$

:3200

:is: $(0)$

:3100

2300

:ifio)

:iviii)

:"sou!

:390)

1(ห)10

f(s)

.$(2 \times) 0$

4300

4.100

$\operatorname{lin} 00$

1600

$4 \pi 00$

4500

$40(0)$

DREDGING CABLE,

CM. DOWN. UR. QM.

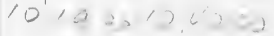

101260010138100

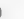




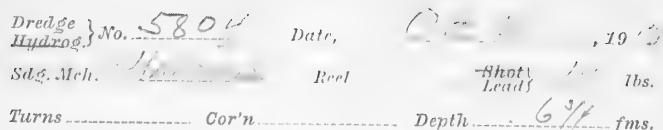

Turns

Cor'n

Amt. Bot'm

Sag. Cup

Depth fms.

Character of Bollom.

Thermom. $\left\{\begin{array}{l}\text { Mater } \\ \text { Xis. Cor'n }\end{array}\left\{\begin{array}{l}\text { Instr. } \\ \text { Stem. }\end{array}\right.\right.$

Bottom Reading

Cor'd Temp.

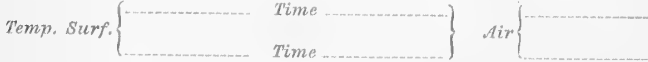

Density: Surf.

fms.

Wind: Direction

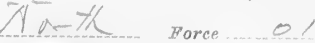

Baromet. $\{0.26$

Amt, of

State of

Weather

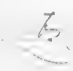

Direction and fforce
of Current. $\left\{\begin{array}{l}\text { Surface } \\ \text { Bottom }\end{array}\right.$

ricle

General Locality

Tosition

Chast used: Mo.

$\pi d ' n$

1)rill: Dirention

Dislunre

Depth of Haut . C

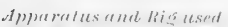

i.
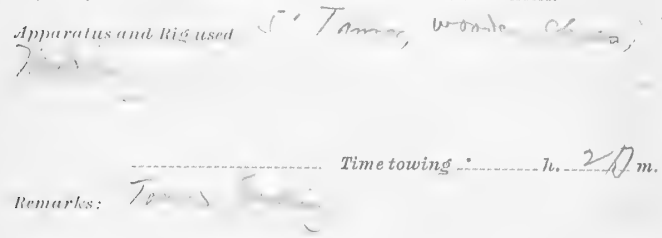

Time towing : 7.27.

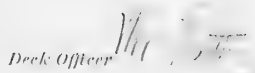


$11-1023$

SOUNDING W1RF.

M. Down. UI.

III

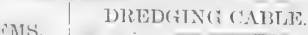

TM. Dow. UT. Z M.

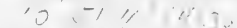

? $10=3,13 \%$

$2(\mu)$

300

.100

50

(i) (10)

i0)

silue

(M)

1000

1100

$12(3)$

1500

1400

1500

1600

1500

$1 \times(10)$

1900

2000

2100

$\sin 200$

2300

$2 \cdot 100$

, 500

$20(0)$

2avo

ㅈavi

$59(0)$

::(10)1

$\$ 3100$

$: ?, 900$

:3:30)

:3106)

3ivolo

:i(i)o

:1700

:3800

$: 39)(0)$

(1)(1)

1100

. 1200

$4: 300$

1. 100$)$

1500

4600

.1700

4800

4000 

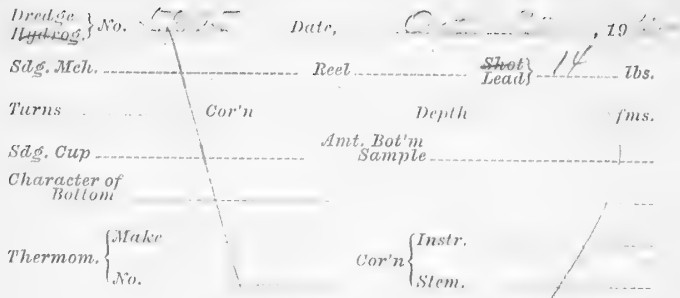

Bottom Reading Cor'd Temp.

Temp). Surf.

Tim.

Tim,

Nensity: Surf.

Wind: Hiretion.

moromel. $30.255 \begin{aligned} & \text { Mmtiof } \\ & \text { ilomits }\end{aligned}$

Weather ?

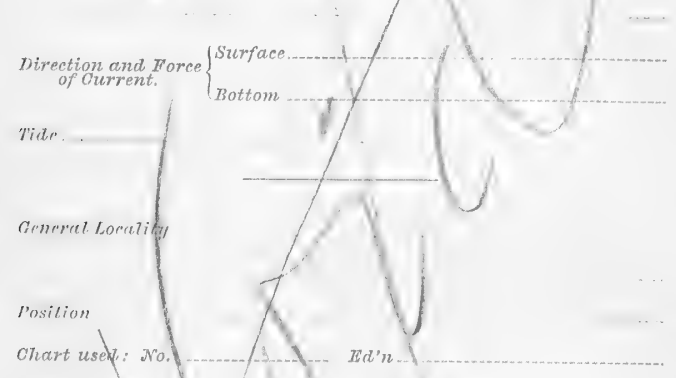

1)rift: Dirention Depth of Hards.

Apparatus atmation used

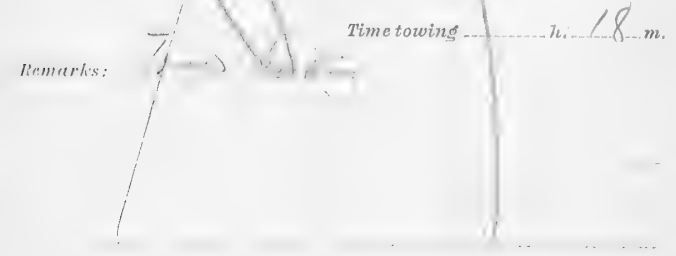


11-102:

SOUNDING WIRE.

DIRLDGINA CAISIS.

M. Down. U1. MI.

KMIS.

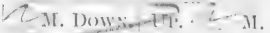
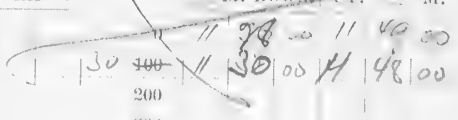

(30)!

(1) (1)

$\mathrm{i}(1) 0$

(ii)

ifo)

Si(1)

() $(1)(3)$

$100(0)$

$11(x)$

1200

1300

1400

150)

1600

$1 \% 00$

1800

1900

?2000

2100

2900

2:30)

$\because 100$

$20(0)$

$\therefore(100)$

2901

3800

:!ou!

$:(0)(x)$

:8100

:*2010

aniter

3100

:3010)

:(i)

सi(i)

$\because 3()()$

:3100)

f(1)(1)

.1100

1200

1:100

f. $1(1)$

- IF)

f(i) ()

. 1 (i)

4800

4!)(1) 


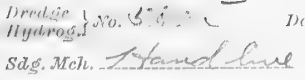

Thrns _ Cor'n

Silst. Cup

Character of
Botioni

thermom. $\left\{\begin{array}{l}\text { Make } \\ \text { Nos. }\end{array}\right.$

Bollom, Readitls

Tem) Surf. Sus. \{

Time

Jati,

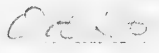

, 10

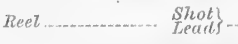

ibs. Amt Bot'm

$$
\text { Cor'n }\left\{\begin{array}{l}
\text { Instr. } \\
\text { stem. }
\end{array}\right.
$$

Vor'd Trm).

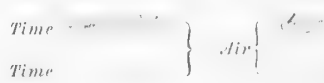

fims.

Density: Surf.

$0 \quad-1$

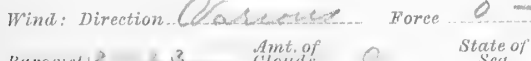

Baromets . \& 3 Aintof

sere

Weather

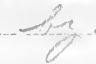

Direction and Foree
of Current. $\left\{\begin{array}{l}\text { Surfuen } \\ \text { Dinfom }\end{array}\right.$

Tide.

General tocatily

\section{Position}

Charet usert: Ao.

En'n

Drïft: Direction Distance

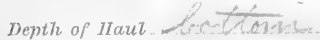

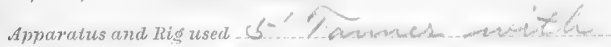
$-2+2+c+c+\infty y$

Time towing 7. 2.? $m$

licmortis: 
SOUNDINT: WVIRE.

M. 1) OWN. UP. M.

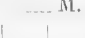

FNS

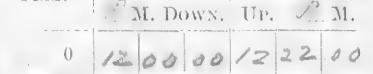




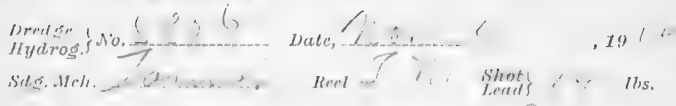
Sill? ciup Sirmpli

Character of

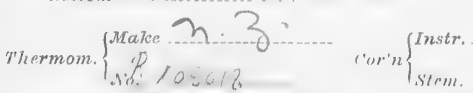

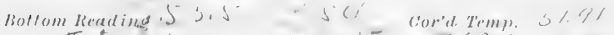

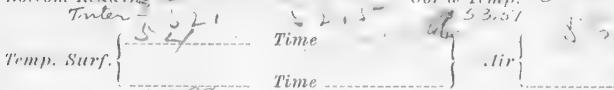

llensily: Surf.

$f m s$.

Wind: Direction Force $6-1$

Baromet.

Ant of 2 State of
Clouds

Heallher

Direction and Horce
of Current.

Tiile

Gineval touralit!

\section{P(s)ilion"}

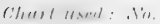

Eil'

Drift: Direction

Distance

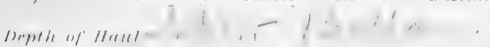

Anparatus and kie used Y' ; i.

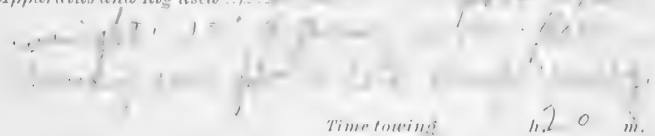

Limmirk: :

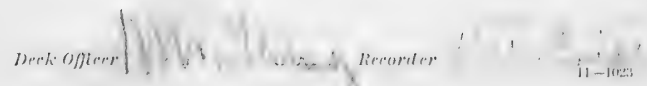




\section{SOUNDING WIRE. \\ - HM. Down. UM. .... M.} 10.4000

FMS.

DREDGING CABLE.

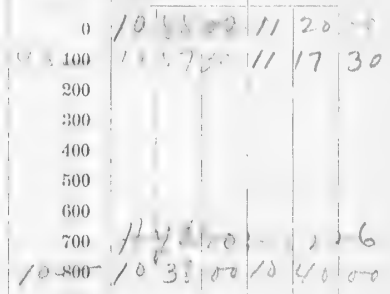

900

1000

1100

1:200

1300

1.400

1500

1600

1700

1800

1900

2000

2100

2200

:

2100

3500

2600

2700

2800

2900

3000

3100

$: 200$

$\$ 300$

3100

3500

$: 360$

:300

3800

$: 3900$

4000

4100

.1200

4301)

1400

4500

4000

4700

4800

1900 
Dredgertio.

Date,

1

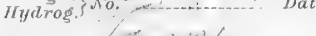

1

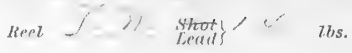

Turns

$\operatorname{Cor}^{\prime} n$

Depth ....

fins.

Sids. Cup

Amt. Bot'm

Character of

Bottoms

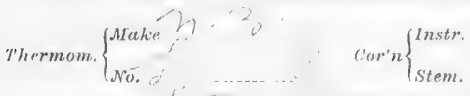

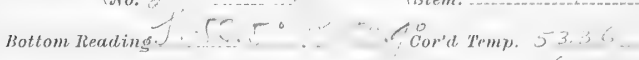

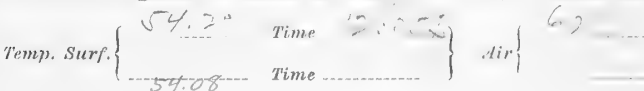

Density: Surf.

fins.

IFind: Direction.

Force

Baromet. 30.27 Alout of

$0-1$

Weather

louds ... 2

State of

Direction and Force
of Current. $\left\{\begin{array}{l}\text { Surface } \\ \text { Bottom }\end{array}\right.$

Till

Generul Loculity

\section{Position}

Gharl Hseal: Aro.

I't'

I)rifl: Direstion

Jislanie.

nepth of Haut of $l_{2}^{3} ; \ldots$

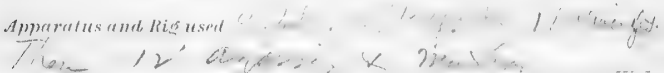

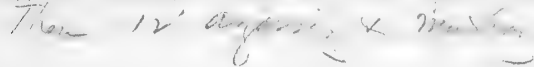

Timn lontus

h. .... $m$.

Remartis:

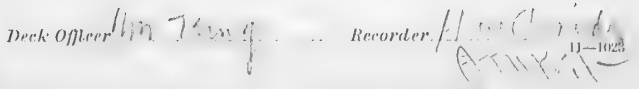


SOUNDINE WIRE.

AM. Down. U1... I.... M. 115330120510

301001212201234 do

300

400

500

(600

700

800

900

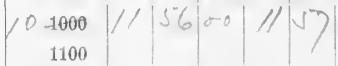

1:200

1300

1400

1500

$1(600)$

1700

1800

1900

2000

2100

2200

$2: 300$

$\therefore 400$

2500

2000

2700

2800

2900

:3000

3100

$3: 00$

3300

3400

3500

$\because 600$

$: 3700$

3800

3900

1000

4100

4200

4300

4400

.1500

4600

1700

1800

4900

DREDGING CAIBLE

it M. DOWN. UP. IN,

121100123520

- 1
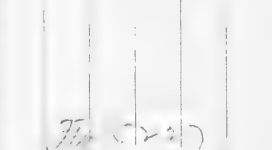

00 
1) reatin ino. 5808 Hentros: Wo. Date, Sels. Mch. Reel

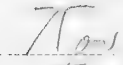

\section{Ticrns}

Cor'n

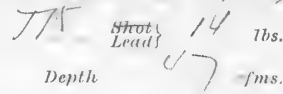

Sd.g. Cup

Amt. Bot'm

Sample.

Charact

koffom

Therpfiom. $\left\{\begin{array}{l}\text { Males } \\ \text { No. }\end{array}\right.$

Boltom Reading

$$
\text { Gorn }\left\{\begin{array}{l}
\text { Instr. } \\
\text { Stem. }
\end{array}\right.
$$

Cor'd Trmp.
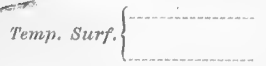

Time

Time

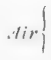

Densily: Surf.

Wind: Direction.

fims.

Buromet. $=\frac{30.25}{76}$

Weather

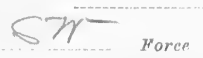

Ant of clouds

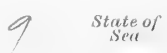

Direction and Force
of Ourrent. $\left\{\begin{array}{l}\text { Surface } \\ \text { Bottom. }\end{array}\right.$

Tide:

Generat Locatily

\section{Position}

Cheret used: : No

$K t^{\prime} n$

Drift: Niretion

Distanee

nepth of Haut 7 ate

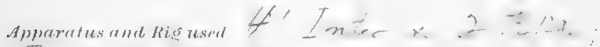

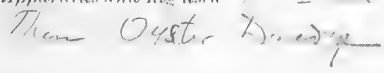

Time towins

n. . . . m.

Remurk:

neck opleer i... - - Rexorler. (-) 
SOUNDING WIRE.

a M. Down. U1. (OM. 1 प6: 0, 1, 18.

$0 y \leq 4$

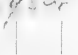

FNS.

DREDGING $\div$ CABTLE.

7 Mr. Down. Up. IT Mr.

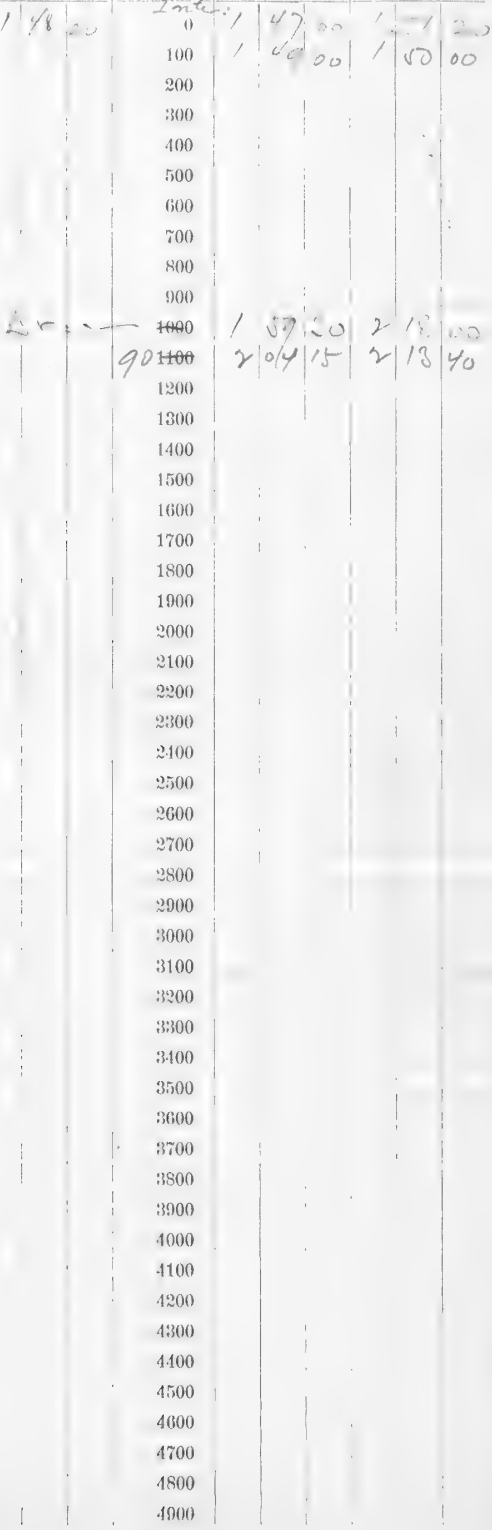




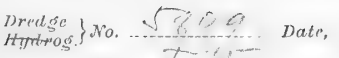

Sils. Meh.

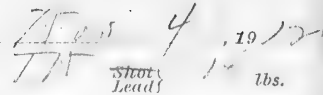

Turns

Cor'n

Depth

fims.

Salo. Oup

Ant, Bot'm

Character of

Rotlom

Thermom. $\left\{\begin{array}{l}\text { Hake } \\ \text { No. }\end{array}\right.$ Oor'n $\left\{\begin{array}{l}\text { Instr. } \\ \text { Stem. }\end{array}\right.$

Bottom Readins

Cor'd Temp.

Temp. Surf. \{

Time

Density: Surf.

Time

$\{\operatorname{Air}\{$

Wind: Direction

fins.

Baromet. 30.2.5 $\begin{aligned} & \text { Amt of } \\ & \text { Cromels }\end{aligned}$

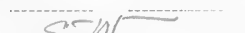

Weather

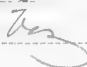

Direction and Force $\left\{\begin{array}{l}\text { Surfare } \\ \text { of Ourrent. }\end{array}\right.$

Tide

Generel Locality

\section{Position}

Chart used: No.

Ed'n

Drift : Direstion.

Distance.

Depthe of Haut

Apparatus and Ris used . C/5

Remarks: 


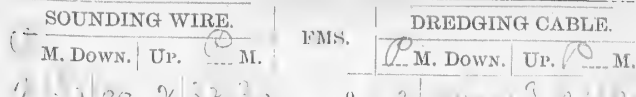
$120 \mathrm{r}$

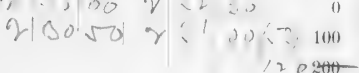

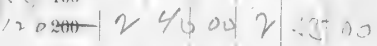

$: 00$

$\$ 00$

500

(i)

\%0)

800

9)(0)

1000

1100

$120)$

1300

1.100

1500

I (i0)

1700

1800

1900

2000

2100

2200

$2): 30(0$

2100

:)

2600

$2 \% 00$

2500

2900

$: 2000$

3100

$3 \geqslant 00$

:3:300

3.100

3,500

$\$ 000$

$\because \because 00$

3500

3900

1000

$\$ 100$

1200)

1:1000

4400

.1500

4600

4700

4800

.19() 


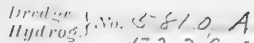

Date,

, 19:

Sus. Mrh. $-\mathrm{S}^{-} 229,4$

Reel

Shoti
letiil

$7 b s$.

Turns

Cor'n

Depth: / $3 / 4$ fins.

Sdg, Cup

Amt. Bot'm
Sample

Character of Bottom

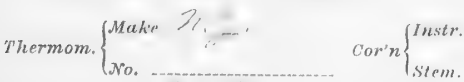

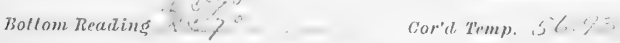

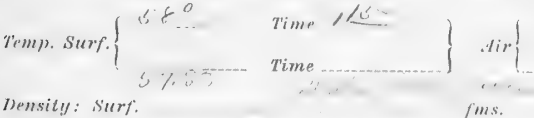

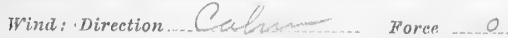

inomet. $2 . . \quad$ Antiof 2 State of S

Weather .......

Direction and Horce
of Current. $\left\{\begin{array}{l}\text { Surfase } \\ \text { Bottom }\end{array}\right.$

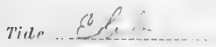

\section{Generat Locality}

\section{Posilion}

Cihurt usel: : win

$\mathrm{Ed}^{\prime \prime \prime}$

Drift: Direction

Distaince

Depth of Haut

better.

Apparatus and Rig used.

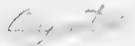

9

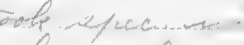

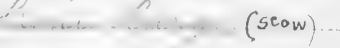

Time towing h. $m$.

Rinmortis

B. 
SOUNDING WIRE.

P. Down. UP. $\infty$ M.

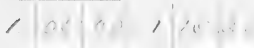

100

200

$\because 3()$

.100

500

(i)

$\div 0(1)$

sion

()()

1000

110()

$1:(90)$

1800

1.100

1500

1600

1700

1800

1900

5000

2100

2200

2:3(0)

2400

2500

2600

2700

2800

2900

3000

$\$ 100$

$: 3200$

33300

3400

3500

$: 360$

$: 3 \% 00$

3800

$\$ 3900$

1000

4100

1200

$4: 300$

1100

4500

$4600)$

1700

1800

. 9000

DREDGING CABLE.

M.
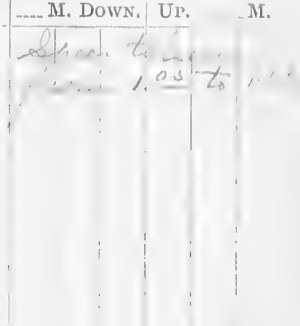

$x^{2}$ 
Turns

Cor'n

Depth: 1/42 fims.

Sds. Cup Amt. Bot'm

Character of Bottom

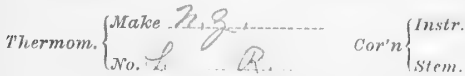

Botlom Reading

Gor'd Trmp, 56.18

Temp. Surf. $\left\{\begin{array}{l}-157.3^{0} \\ -57,73\end{array}\right.$

Time

Time

$\operatorname{sir}$

7)ensity: Surf. fms

Wind: Direction

(1)

Baromet.

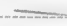

Amt.of 2 State of

Weather

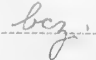

Direction and Force
of Current. $\left\{\begin{array}{l}\text { Surface } \\ \text { Bottom }\end{array}\right.$

Fill"

General Locality

\section{Position}

Chart used: No.

$K l^{\prime} u$

Drift: Direction

Distance

Depth of Haul

Apparatus and Ri@ used

Coten

Remurks: $t$, ....... 
SOUNDINA WIRE.

Q. M. Down.| UP. PM.

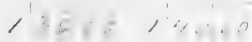

FMS.

M.

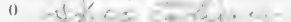

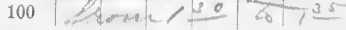

200

:310)

410)

500

(500)

700

800

900

1000

1100

1201

1800

1400

1500

1600

$1 \% 00$

1800

1900

$2001)$

2100

2200

2300

2100

2500

2600

$\approx 700$

ख्य 00

ख्य(y)

$3(000$

\$3100

:3200

:3:300

3400

3900

:3600

:1700

$: \$ 800$

:3900

4000

.1100

.1200

4300

4100

4500

.4600

$17(k)$

.400

.1900 


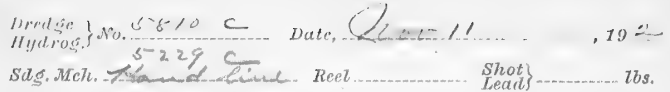

Turns

Cor'n

Depth $1 / 1 / 4$ fms.

Sag. Cup Amt. Bot'm

Character of

Thermom. $\left\{\begin{array}{l}\text { Water Ni jo. } \\ \text { No. }\end{array}\right.$ Cor'n $\left\{\begin{array}{l}\text { Instr. } \\ \text { siem. }\end{array}\right.$

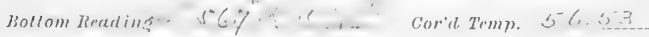

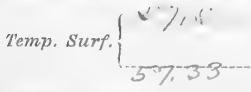

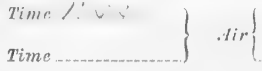

l)wsity: surf:

fins.

Wind: Jirection.

litromel. Alnt of 2 State of Weather ... $\mathrm{b}_{3}$

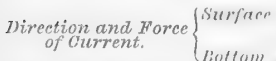

tiule.... $\leqslant / \%$

General Locality

\section{Posilion}

Chart used: No,

E'd'n

J)ifl: Jimertion

Disfaner

Depth of Haul

Apparatus and Rig used

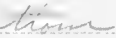

Q2 befiane

Timetowins

i.

$m$.

limmurtes: $C_{0}, \ldots, 1, \ldots$ 
SOUNDING WIRE.

II. Down. Ur. M. $-|43| 40,15-340$
DREDGING CABLE.

FMS.

M. Down. UP. _... M.

200

3001

100

500

(i) ()

i00)

800

(9)(0)

1000

1100

$12(k)$

1300

1400

1500

1600

1700

1800

$1900)$

2000

2100

2200

$2: 300$

2400

2500

2600

2700

2800

2900

$\$ 3000$

3100

:300

33306)

$: 300$

3500

$\$ 360$

$: 3700$

3400

3600

4000

1100

1200

.1300

4400

4500

$4(60)$

4700

4800

4000 


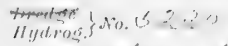

siles. Mrh

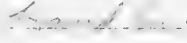

l) le,

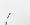

\section{Turns}

Cor'n

IInI

$$
\text { Denth }
$$$$
\text { Amt, Bot'm }
$$

Silis. (cup)

Character of

Whermom. $\left\{\begin{array}{l}\text { Make } \\ \text { Joe. }\end{array}\right.$

Botlom Rearlins

Timp. surf.

r.

Densit!: Surf.

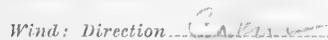
Gor'n $\left\{\begin{array}{l}\text { Instr. } \\ \text { stem. }\end{array}\right.$

C'or'cl T'mm).

Baromet.

Amt. of Clouds.

fills.

Horce 0

\section{State of}

Weather

Direction and Force
of Cument. $\left\{\begin{array}{l}\text { Surfuce } \\ \text { Buttom }\end{array}\right.$

ride'

\section{General Loretity}

\section{Position}

Chart used: No.

Drift: Direction

Distance.

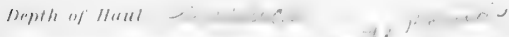

Apporalusand lisigused th

(1)
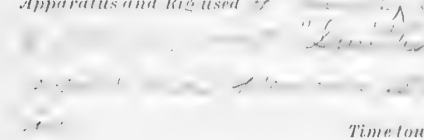

Time fouvins

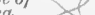


P. Down. UL, D. FMSS.

M. Down. UP. PM.

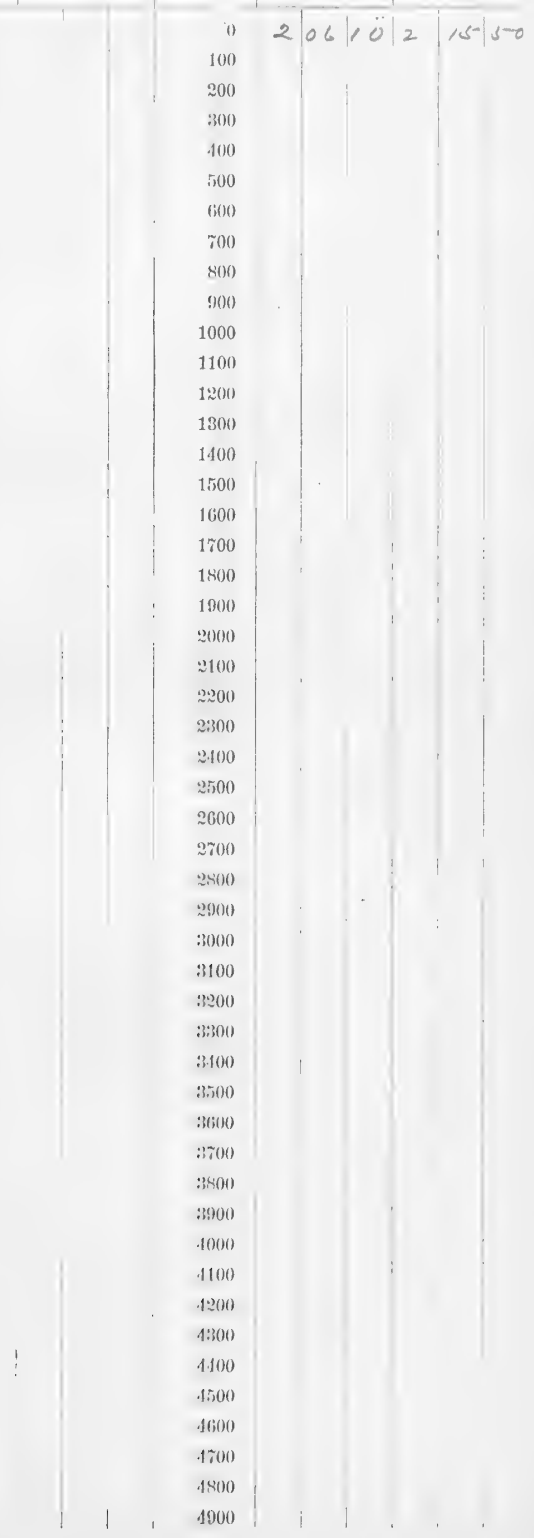


Hydrog tru. $\frac{11-1}{523 /-}$

Sd. Mch. . 2

Date, .... $1, \ldots$

, $19 \cong$

Turns

Reel

Shot

lbs.

Sils. (u)

Cor'n

Depth: L:

fins.

Charucter of

Thermom. $\left\{\begin{array}{l}\text { Nake, }, i, j \\ \text { Nis. }\end{array}\right.$

Botlom Reratins

$$
\text { Amt.Bot'm }
$$

$$
\left.\operatorname{Trmp} \text { Surf. }\left\{\begin{array}{ll}
5-y, j & \text { Time '? } 0^{\prime} \\
5 y, 53 & \text { Time }
\end{array}\right\} \operatorname{\text {Nir}}\right\}
$$

Drnsity: surf.

fins.

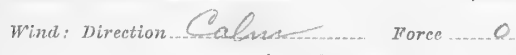

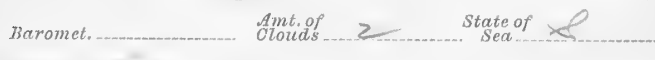

Heather

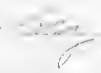

Direction and Froree $\left\{\begin{array}{l}\text { Surface } \\ \text { of Current. }\end{array}\right.$

Tiut $\ldots+\cdots$

General Locality

\section{Position}

Chart used: No. Nd'n

Drift: Direction

Distance.

Deputh of Haut.

Apparatus and Rig used

,

Time touvins

h.

$m$.

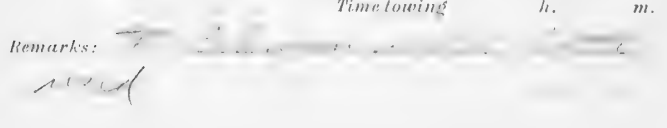


11-1029

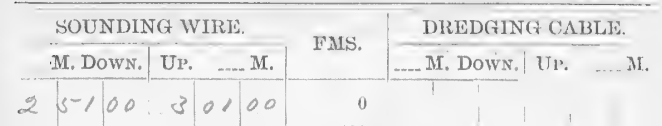

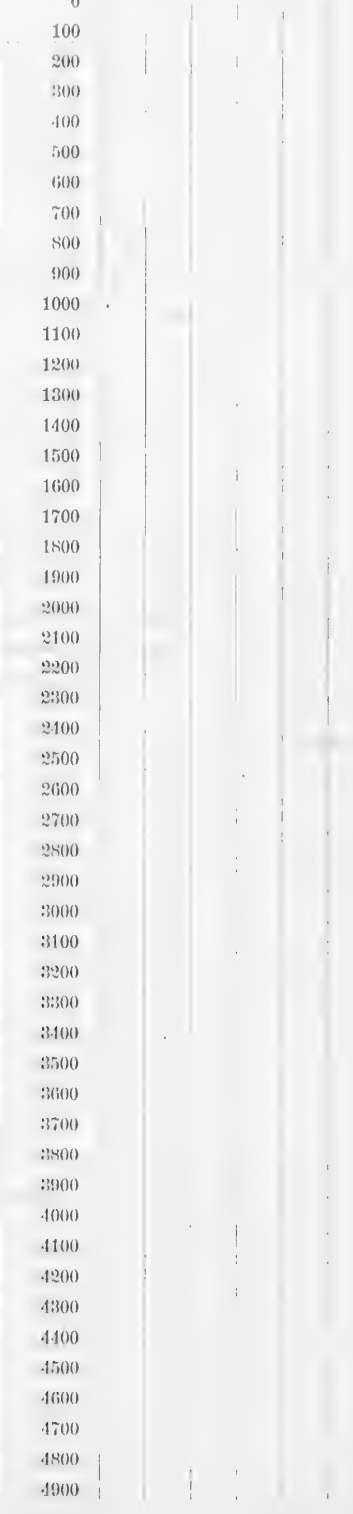




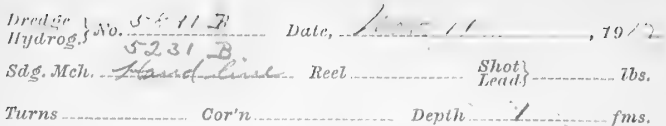
Sits. $6 u p$
Amt. 3 sot'm
Character of Bottom
Thermom. $\left\{\begin{array}{l}\text { Make }>, \ldots, j \\ \text { No. }\end{array} \quad\right.$ Cor'n $\left\{\begin{array}{l}\text { Instr. } \\ \text { Stem. }\end{array}\right.$

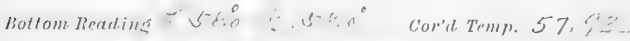

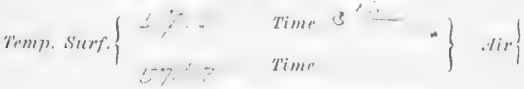

Density: Surf. fons.

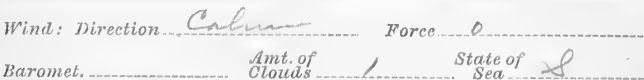

Weather . . n...........

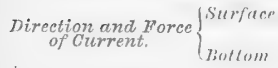

Tiale, eff

General Lorality

Position

Chart used: No.

$n d l^{\prime} n$

Drift: Direction Distance

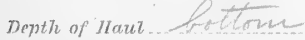

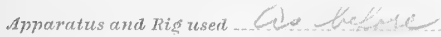

i

.... Time towing 7. $m$

Remurlis:
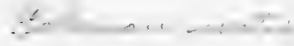

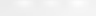


$11-1023$

HOUNATHC WIRE.

M. Down. UP. $\mathrm{M}$.

FMS.

DREDGING CABLE.

$3|02| 20$ 3/ 3120

M. Down. UP. MI.

100

200

$\$ 300$

400

500

6it)

ร00)

$8(1)$

(9)(0)

1000

1100

1200

1300

1400

1500

1600

1700

1800)

1900

2000

2100

2200

2300

2100

2500

2000

2704)

$28(x)$

$290(x)$

$: 000$

$\$ 3100$

$: 3200$

:3300

3.100

3.5.01)

:3600

$: \%(100)$

:800)

:(6)

4000

4100

4200

4300

4400

5000

f(io)

5000

4800

1900) 
Dreage 3 Hodrog, sif/C

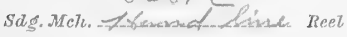

Date,

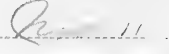

, 19

\section{T'urus}

Cor'n

7) cyll

Shot Zbs.

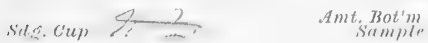

Character of Boltom

Thermom. $\left\{\begin{array}{l}\text { Wake } \\ \text { No. } \alpha \text { Cor'n }\end{array}\right.$ Inster.

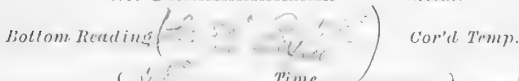

Temp. Surf. $\left\{\begin{array}{ll}t: & \text { Time } \\ & \text { Time }\end{array}\right\}$ tir $\}$

$$
\therefore \% \ldots=\text { fins. }
$$

1)ensit!l: surf.

Wind: Direction

Force ......

Boromet

Amt. of 2

State of Sea

Weallutr<smiles>C=CC=CC</smiles>

Direction and Force
of Current. $\left\{\begin{array}{l}\text { Sufufuen } \\ \text { bottom? }\end{array}\right.$

Tiate...se

General Locality

\section{rosition.}

Chart used: No.

lf d'n.

H)ift: Dirrefion

Distumen

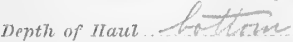

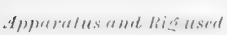

Time towing.

h.

$m$.

Remterlis:

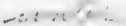

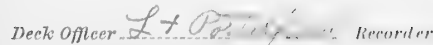

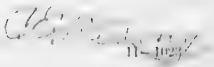


SOUNDINA WIRE.

(. Mr. DOWN.| Uน. M.

FMS.

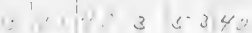

DREDGING CABLIE. M.

200

$: 00$

.100

500

(i) 0

700

800

900

1000

1100

1:00)

1300

1400

1500

$16(60)$

1700

1800

1900)

2000

2100

2200

$2: 300$

2.100

5500

2000

2700

2800

2900

3000

$\$ 3100$

3200

:3:304)

3100

3800

$\therefore 660$

:3ro0

$: 3800$

3900

1000

1100

.1200

4300

4.100

1500

4600

4700

4800

.1800 
$\left.\frac{\text { Dnedige }}{\text { Hydro }}\right\}$ No 152,2

Sd g. Meh.

Turns Corn
Date, Reel Shot Depth 1114 Amt Bot'm tbs.

, 19 fms.
Sols. Cup

Character of

Bot tom

Thermom. $\left\{\begin{array}{l}\text { Make" } \\ \text { No. }\end{array}\right.$

Bottom Reading

Temp. Surf. \{

Density: Surf.

Wind: Direction

Baronet.

Ant. op

Weather be
$\operatorname{Cor}^{\prime} n\left\{\begin{array}{l}\text { Instr. } \\ \text { Stem. }\end{array}\right.$

Cor'd Temp.

Time

Time

Force

State of rims.

$\{\operatorname{lir}\}$

Direction and Force
of Current. $\left\{\begin{array}{l}\text { Surfer } \\ \text { Bottom }\end{array}\right.$

Tile +Le .tin.

General Locality

Position.

Chart usenet: Non.

Ext' '

Drift: Direction

Distance

Depth of Haul Lenblaces.

Apparatus and Rigs used

Time towing

n... $0 . . n$.

Remarks:

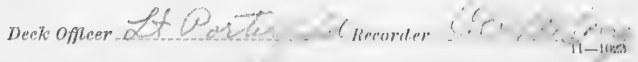


SOUNDING IVIRI.

$\sigma^{\prime}$ M. DOWN. U'. Fns.

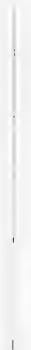

$0|\&| 0400|\psi| 14 \mid 0.0$

100

200

:301)

400

500

(i)()

700

800

()(1)

1000

t100

1200

1300

1.100

1500

1600

$1 \% 00$

$18(60)$

1900

2000

$\approx 100$

2200

2390

$\approx 100$

2500

2600

$2 \pi 00$

2800

2900

3000

$\$ 100$

$3: 00$

3300

$\$ 100$

33,000

$: 300$

:3\%01

$: 3800$

$: 900$

1000

$\$ 100$

1200

(1:30)

4.100

4500

Ifion

.1700

1800

(19)(10)

DREDGIN : CAIBT, 


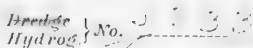

J) at?

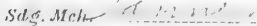

Reel. , ! Short?

Ths.

Turns

Cor'n

Amt. Bot'm

$\sin ; \cdot(u)$ Sample

foms.

Character of Botfom

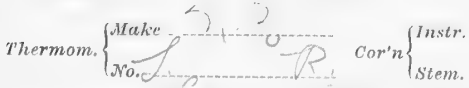

liottom Reading. I

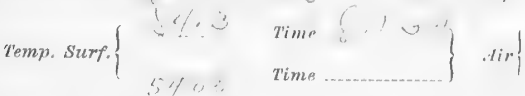

lensity: Surf.

fills.

Wind: Direction.......

Foref $8-2$

Raromet. 30,2 a $\begin{aligned} & 3 \text { Amt.of } \\ & \text { Clowds }\end{aligned}$

State of

(1)

Direction and Force
of Current. Sultime

Tide

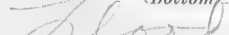

General Lorality

Position

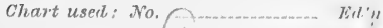

Drift: Direction. 人) Distance

Depth of Haut $\mathrm{Sul}$ :

Apparatus and Ris used th
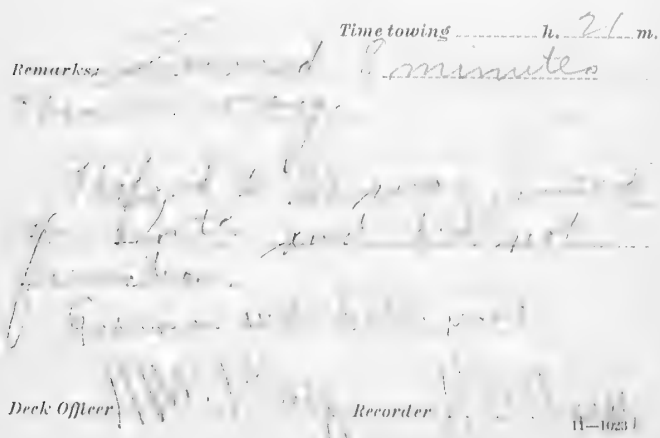
, SOUNDING WIRT.

M. INowN. UT. M.

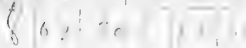

FMS.

DREDGING CABLE.

(1)

I(t)

200

(3)(1)

.100

500

(it)

700

800

!)(1)

1000

1100

$1: 200$

1300

1400

1500

1600

1700

1800

1900

2000

2100

200

$2: 300$

2100

2.500

$\because 600$

2ร00

2500

2900

3000

$\$ 3100$

$\because 200$

:3300

3.100

3500

$: 3600$

$3 \% 00$

$: 3800$

3900

.1000

$\$ 100$

1200

43000

4.100

1500

di:

1700

.1500

4900

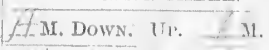

$\int 10300$
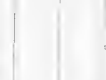

.
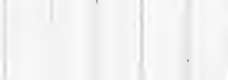

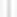




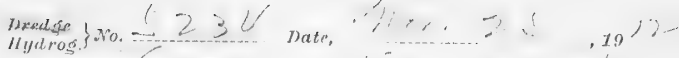

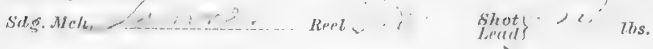

$$
\begin{aligned}
& \text { Thrus dor'n Jepth fins. } \\
& \text { Silg. C'up) Amt llot'm }
\end{aligned}
$$

Churacter of Bottom

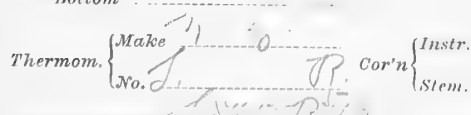

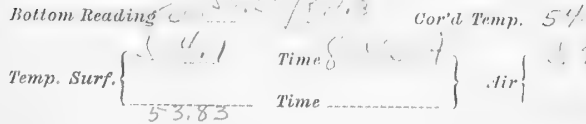

Density: Surf.

Wind: Direction........

Buromet. $30.1 \% 9$ Amtof 9 louds..

Weather ...

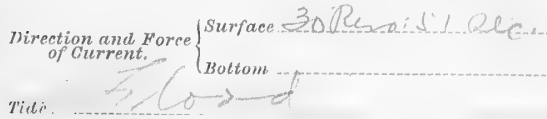

\section{General. Lorality}

\section{Position}

Chart uspol : No EII'n
Drift: Direction. Whend Distance

Depth of Haul

Apparatas and Ris laseal

\section{Remurk's:}


SOUNIDING WIRE.

M. Down. Ur. Ц̈M.

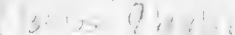

FMS.

DIREDGIN(* ('ABLLE.

H M. Down.l UP. jA M.

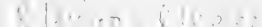

100

200

300

100

500

(i)0

700

800)

900

1000

1100

1200

1300

1400

$1500)$

1600

1700

$1 \mathrm{R} 00$

1900

2000

2100

$\approx 200$

$2: 300$

2100

2500

2600

$2 \pi 00$

2800

2900

3000

3100

$33: 00$

3:300

$\$ 400$

3500

3600

$: 3700$

$: 800$

3900

4000

4100

4:00

4:300

4.100

4500

4600

$\sqrt{7} 00$

4800

4900 


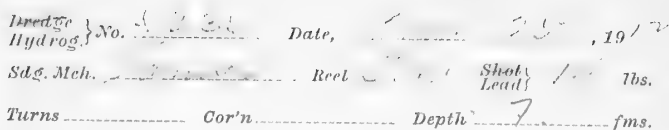

Sas. Cup

Amt Bample.

Character of

Bottom

Whermom. $\left\{\begin{array}{l}\text { Make h. 2 } \\ \text { No... }\end{array}\right.$

Bollom Readings ... Cor'd Trmp, 53.98

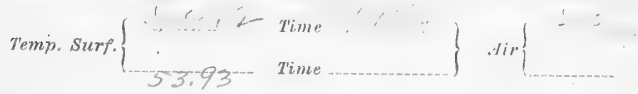

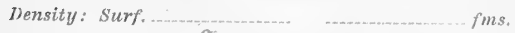

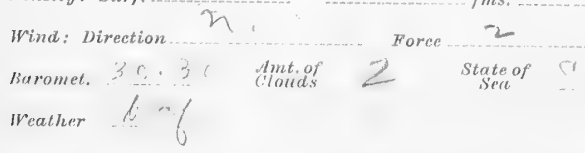

Direction and Force
of Current. Surface $2,1, \ldots, \ldots, \ldots$

Tiile: .

\section{General Loeatily}

\section{fosition}

Chart used : No

IEl'n

Drift: Direction ( $, \vdots, \ldots$, Distance

Depth of Haut

Apporatus a mol ligig used
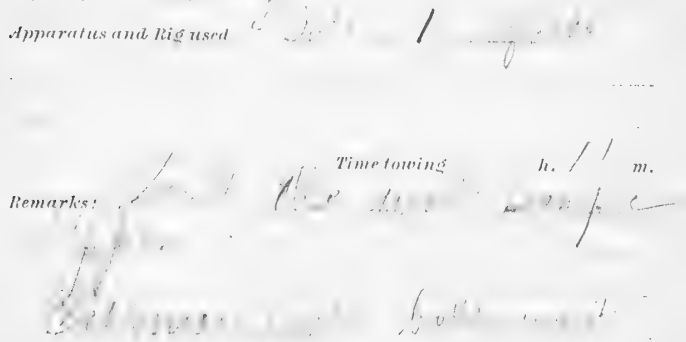

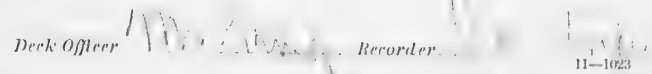




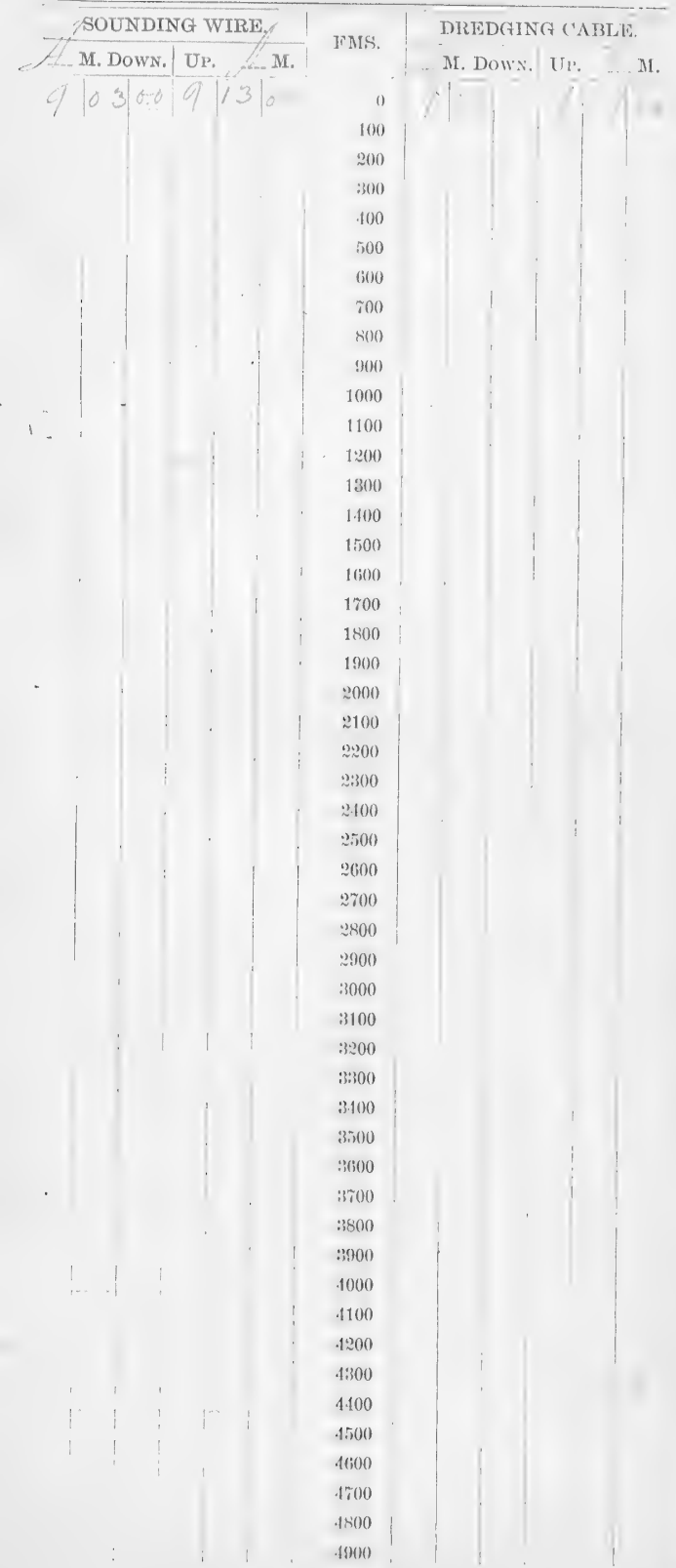




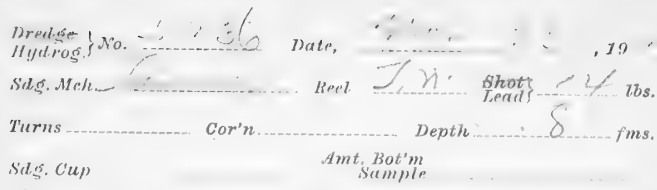

Character of Bottom.

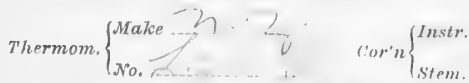

Bottom Reading $\quad$ ?

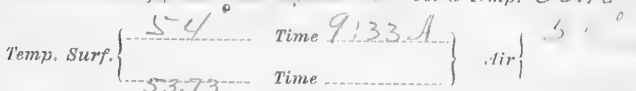

Density: Surf. fims.

Wind: Direction.

Forte

Baromet. 30.3 .9 Amtiof 8 Stoute of

Weather $\varliminf_{-\infty}$

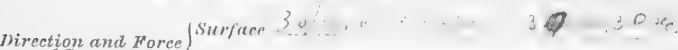
of Ourrent.

l Botlom

Tillr

\section{General Locality}

\section{Position}

Chart used : Atis.

Fin'

Drifl: Inirertion

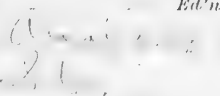

Dopth of $\mathrm{Hant}$

Apparatus and Ris ased

Distance 
SOUNDING WIRE.

A. DOWN. UP. M.

DREDGING CABLE.

$9.22 \mid$ so: $9|3.2| 0$.

FMS.

UP. A. M.

00

200

;300

400

500

(\$0)

700

800

900

1000

1100)

1200

1300

1400

1500

1600

1700

1800

1900

2000

2100

2200

2300

: 400

2500

2600

2700

$2 \times 00$

2900

3000

$: 3100$

3200

33300

3400

3500

3600

:3)

3800

3900

.10010

4100

1300

4:300

4.100

aivol

4600

$4 \% 00$

4800

4900

q) 2300

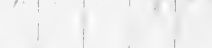

$+$

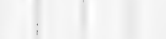




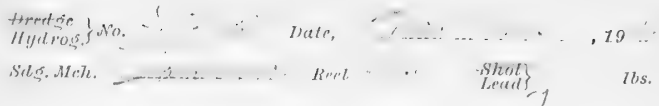

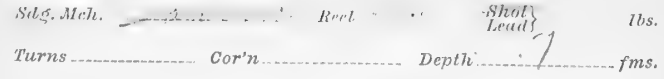

Sidg. Cup

Amt. Bot'm

Character of Jioltom

Whermom $\left\{\begin{array}{l}\text { Malee } \\ \text { No. Oor'n }\end{array}\left\{\begin{array}{l}\text { Instr. } \\ \text { Stem. }\end{array}\right.\right.$

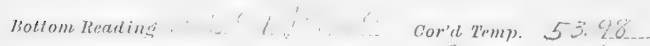

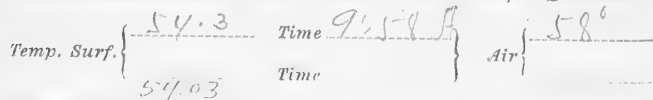
l)ensity: stor\%.

fins.

Hinll: Jirection

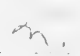

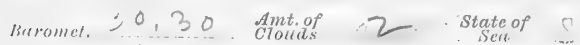
Weather

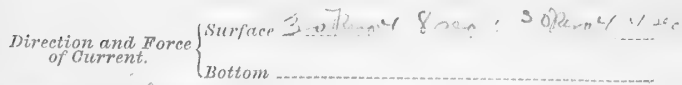
Tills

\section{General Locatity}

\section{Posilion}

Chart used: No Fin'n

Drift: Direction... Distance Depth of Haut. Sue

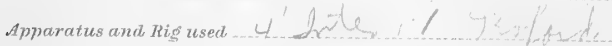

lemerlis: :

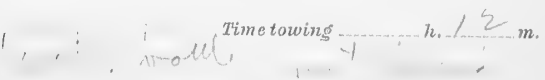


11-1023

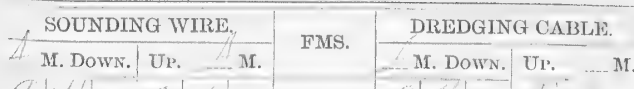

46600.4560

M.

100

200

300

400

500

600

700

800

900

1000

1100

1200)

1300

1400

1500

1600

1700

1800

1900

2000

2100

2200

2300

2400

2500

2000

2500

2800

2900

$\$ 3000$

$\$ 100$

:3200

$3: 300$

3400

3500

$: 3000$

3700

3800

$: 3900$

.1000

.1100

4200

4300

4400

1500

4600

$.1 \% 00$

4800

4900 


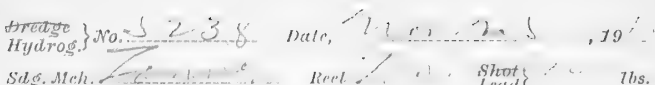
sils, Mch.

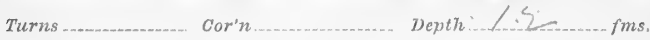

Sisls. $C u$ (Amt, Botim

Character of Bollom

Thermom. $\left\{\begin{array}{l}\text { Makn } \\ \text { No, }\end{array} \quad\right.$ Cor'n $\left\{\begin{array}{l}\text { Instr. } \\ \text { stem. }\end{array}\right.$

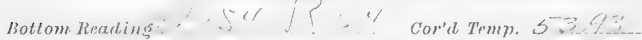

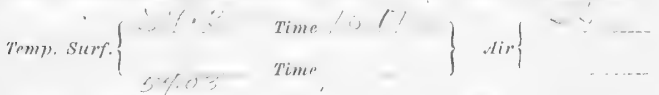

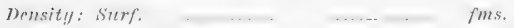

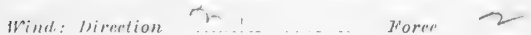

Jiaromet. 30,30 Amtof 4 State of S

Weather....

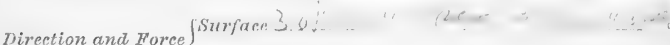
of Current. $\{$ Bollom

Ticke

Generct Lorrality

I'osition

Chart usinl: No. Hill

Drift: Direction. Distanee

Deptic of Haut...s.

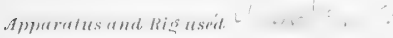

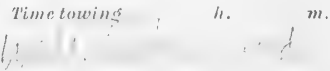

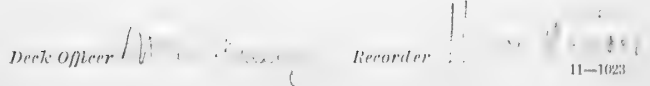



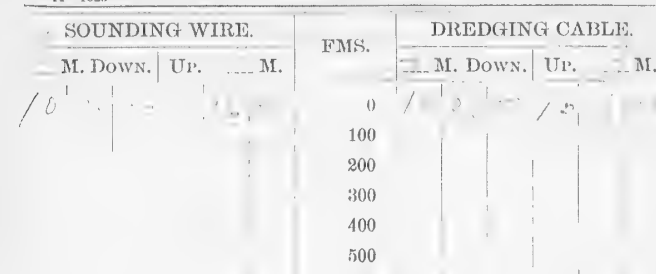

600

700

800

900

1000

1100

1200

1800

1400

1500

1600

1700

1800

1900

2000

2100

2200

2300

2400

2500

2000

2\%00

2800

2900

3000

3100

3200

3300

8400

3500

3600

3700

3800

:3900

4000

4100

4.200

4800

4400

4500

4600

4700

4800

4900 


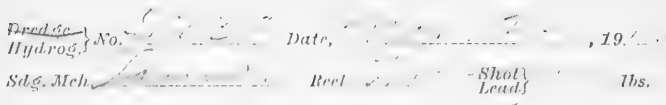

Turns

Cor'n

$$
\text { Denth:... } 20
$$

fins.

Sid, $\{$. $C u]$

Amt. Bot'm

Character of

Thermom. $\left\{\begin{array}{l}\text { Make } \\ \text { No, }\end{array}\right.$

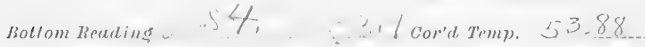

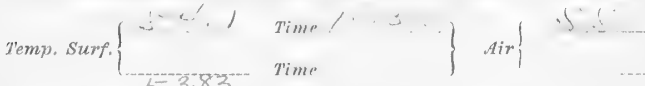

$$
5-3.83
$$

fensity: Surf.

fins.

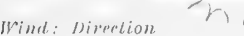

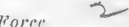

Baromet. 30,29 Amtof State of

Weather ....t.

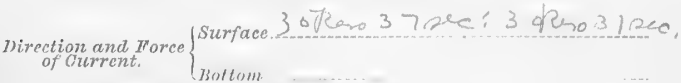

Tills

\section{General Lorality}

Position

Chart used: No.

$\pi d^{\prime} n$

Drift: Dirertion ( + ? is e ..', Distaner

Jepth of Haut...

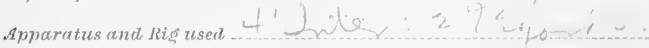

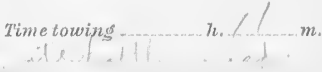


SOUNDING WIRE.

M. Down. Ud. M.

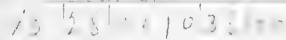
DREDGING CABLE.

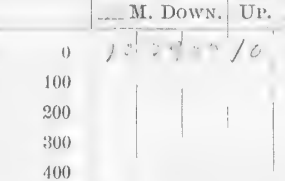

500

(i)

700

800

900

1000

1100

1200

1300

1400

1500)

1600

1700

1800

1900

2001

2100

2200

2300

$\approx 400$

2500

2600

$2 \% 00$

2800

2900

$\$ 3000$

3100

3200

83:300

3400

13.500

$3(600$

$: 3700$

$: 3800$

23000

$4(0) 0$

.1100

1290

4304

4100

1500

4600

$1 \%(00$

4800

4000

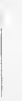

... II.
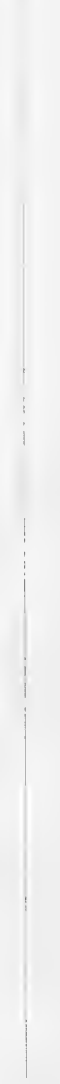


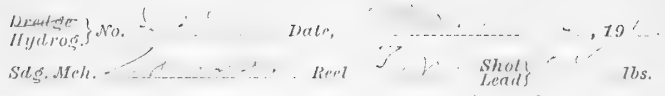

Turns

Cor'n Depth $\ldots 2$ frns.

sile. cur

Amt. Bot'm

Character of

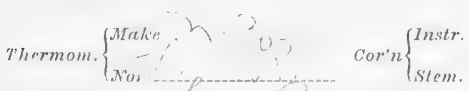

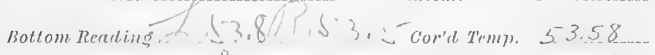

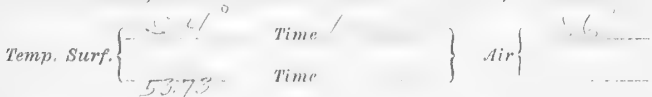

Jusity: surf.

fins.

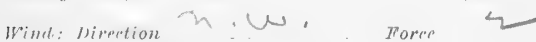

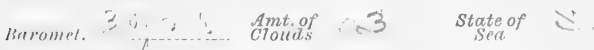

Weather for $\mathrm{C}$.

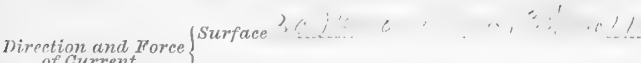
of Current. $\quad$ isultom

Tide

Ceneral. Locatity

\section{Position}

Chart used: No

$\pi d^{\prime} n$

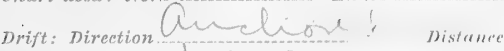

Depth of 11 aul

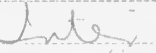

Apparatus and Rio used
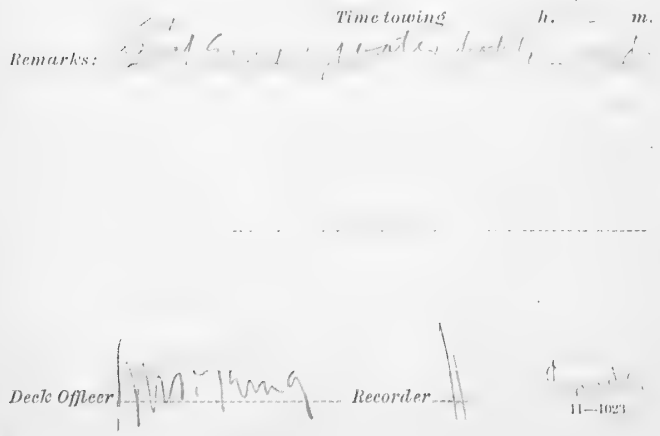
11-1023

SOUNDING WIRE.

$\therefore$ M. Down. U1. … M. F. Fis.

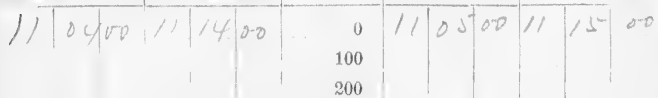

$\$ 300$

400

50

(i)0

700

800

900

1000

1100

12)(1)

1300

1400

1500

1600

1700

1800

1900

2000

2100

2200

2300

2400

(3) $0(0)$

2600

2700

2800

2)( $)(0$

3000

$\$ 100$

3300

\$3300

3100

3500

$: 3600$

स3त( )

3800

$: 3900$

1000

1100

4200

4:300

4400

.1500

A(i)

1700

1800)

41)(x) 


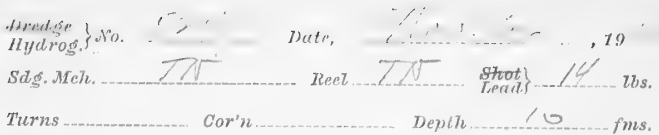

silg. Cup $\quad$ Amt. Jiotim

Character of jottom.

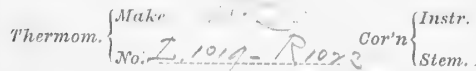

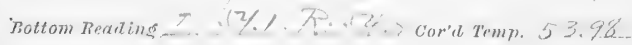

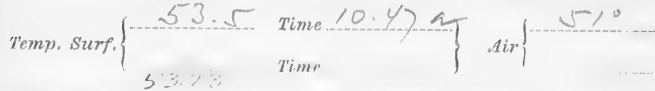

Thplsity: Surf. fms.

Wind: Direction.
Buromel. ? Porce

Weather

$\because$,

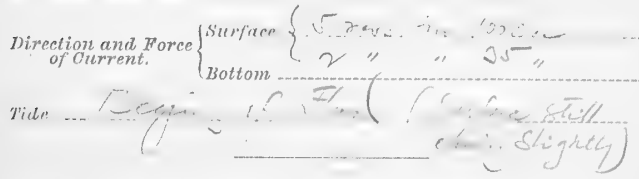

General foratily

Position

Chart used: No, El'ln

1)ifl: Dirention

Distaner:

Deptr of Haut... T nter

Amparatus and kigaseal

$4,7 x+2$ ?

Remurks: 1 Time Lomins

h. $"$ "

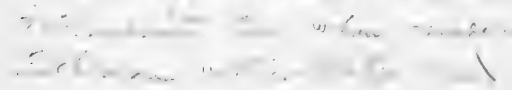

merkofter. 
SOUNDING IVIIRE.

. MI. Dow. U1. ... MI $\therefore, 7.101 \%), 0$
FNS.

DIREDGING CAIBLE,

M. Dow, ITP : M.

(1) $-7 \ldots 11,201$

100

200

:3010

100

500

fion

ז00

800

90

1000

1100

1200

1300

1.100

1500

$16(0)$

1700

1800

1000

2000

2100

2200

$2: 300$

$\because 400$

5000

2600

$2 \% 00$

2800

2900

$: 3000$

3100

3200

\$3900

3100

3.50

3600

:3\%00

$: 3800$

3900

1000

.1100

.2900

(1:10)

1100

4500

4600

4700

4800

.9900 


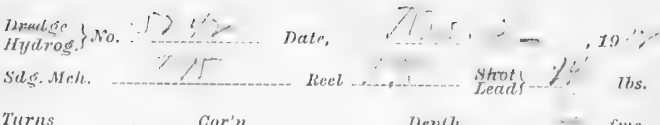

$$
\begin{aligned}
& \text { Turns Cor'n Depith - fins. } \\
& \text { Sils. Cup } \\
& \text { Amt. Bot'm }
\end{aligned}
$$

Chreracter of Bottom

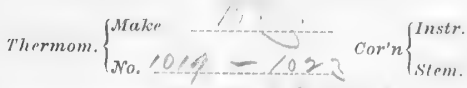

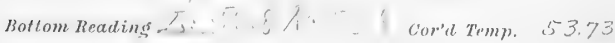

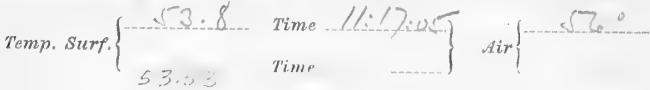

Density: Surf.

fims.

Wind: Direction
Baromet. $\quad$ ? 0 Amt of

Weather .............

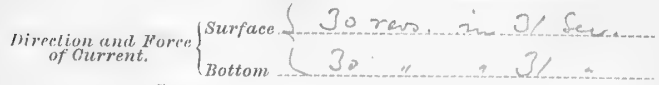
Tiale $2+2,2$

\section{General Locality}

\section{Posilion}

Chart used: : Av.

Drift: Direction

Distanie.

Deptit of Haut .... Tnter.

Apparalus and Rigigused

Timer toutins

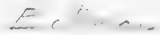

h. $\quad \geq m$

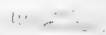


SOUNDING TVIRE,

\#. Dows. Ur. M. $1 / 107$ ar $1 / 1\rangle$. 05
FMS.

DIREDGING CABLE.

M. Dow. I'. M.

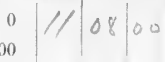

200

300

400

500

(300)

ro(t)

sol)

(9)

10010

1100

1200

1300

1.1100

1500

$1(60)$

1700

18(1)1)

1900

$30(10)$

2100

2000

$2: 300$

$\approx 100$

2500

2000

2700

$\$ \times 00$

2900

$: 3000$

3100

3200

:3300

3100

3300

$: 300$

$: 300$

:1800

3900

8000

.1100

1200

4300

4400

4.500

4600

.1700

4800

$+1900$ 


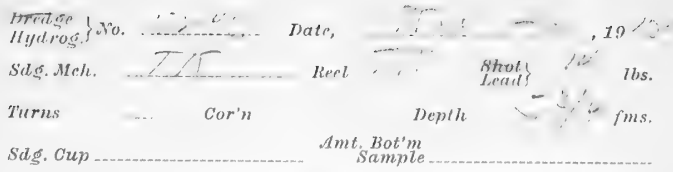

Character of

Botiom

Thermom. $\left\{\begin{array}{l}\text { Make .... } \\ \text { No. }\end{array}\right.$ ('or'n $\left\{\begin{array}{l}\text { Instr. } \\ \text { Stem. }\end{array}\right.$

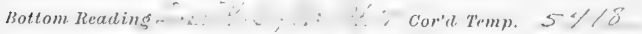

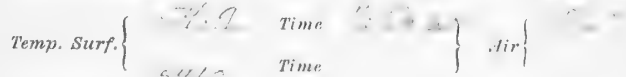

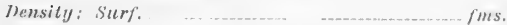

Wina: Direction... ENFE Fren 3

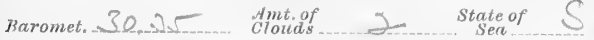

Weather .........

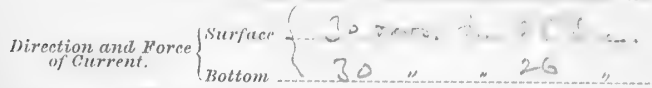

Ticle

ב-

General. Locatity

Position

Chart used: No.

L'l'n

Drift: Direction

Distance

nepth of Haut ..... Tote i

dpparatus and Rizis used c. $=5, k+5)$

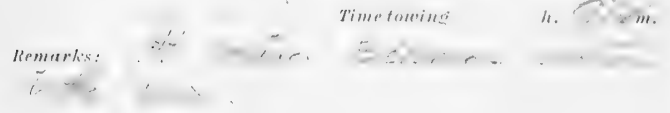

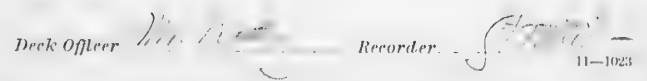


SOUNDING WIRI.

$\because$ M. DOWN. U1. \&... MI. $* 146100|/| 12100$
FMS.

DRFDGINA CABLE.

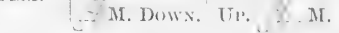

100

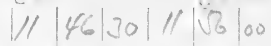

200

$: 3()$

, 100

500

(b) ()

\%00

\$ino

!)

1000

1100)

$1: 00$

1300

1.100

1500

1000

1700

1800

1900

2000

2100

2200

$2: 300$

$\because 100$

500

2000

2 '

2800

2900

3000

3100

$: 300$

$3: 300$

3100

3500

:300

:ะช00

$: 3800$

$\$ 900$

.1000

$\$ 100$

4200

4300

4400

.1500

4600

1700

$+500$

$4900)$ 


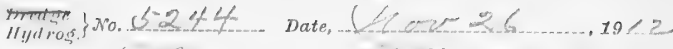

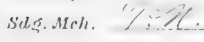

Turns

Sdg. Cup

Character of
Rerl

Cor'n

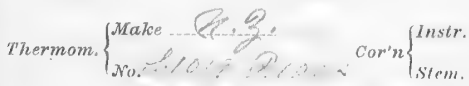

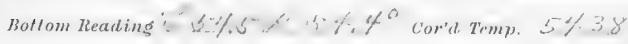

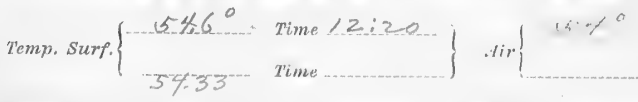

Density: Surf.

fims.

Wind: Direction E... है,

Foree $2=3$

Baromet. 30.24 Amt of

0

stute of

Weather

Direction and Force
of Current. $\left\{\begin{array}{lllll}\text { surface } & 3 & 0 & 1 & 6 \\ \text { nollom } & 3 & 0 & 4 & 4 . .\end{array}\right.$

Tide yेtén

Generat tocality

Position

Chart used: Ano.

Ea'n

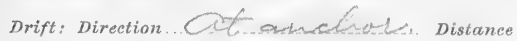

nepth of Hinut doveter.

Imparalus and Ris used

$\therefore, \therefore$,

at ane that

Remurks: Timetomins h. Is m.

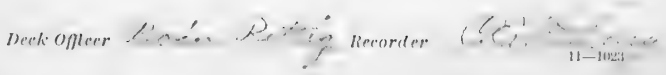


SOUNDINA WIRE.

$\checkmark$ MI. Down. UT, II. $12.1 \%, y,=1 \%$
FMS.

DREDGING ('A BLE.

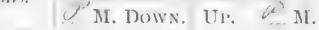

()

100

200

:30()

.100

500

(100)

\% 00

$8(1)$

900

1000

1100

1200

1300

1400

1500

$1600)$

1700

1800

1900

2000

:100

$\approx 200$

$2: 3(1)$

$\therefore 400$

2500

2600

$2 \pi(10)$

2500

2900

$: 3000$

3100

:3:00

:3:300

3100

35.500

:3600

:3700

$: 800$

$3: 3000$

.1000

1100

4200

$4: 300$

4100

4500

4600

4700

4800

49010
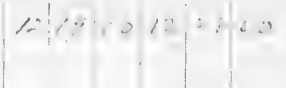


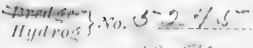

Sils. Mch.

Tiurns

Cor'n

\section{Date, wi f:? 6 \\ $10<2$

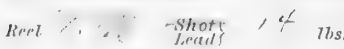

Depth $\quad$ ims

Sids. Cup

Amt. Bot'm

S(xm)le

Character of

Bottom

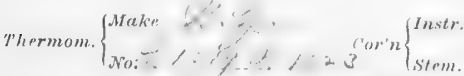

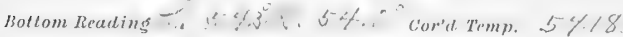

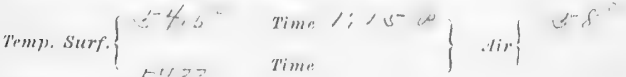

$$
5132 \text { Time }
$$

Density: Surf.

fins.

Wind: Direction

rorer $=-$ is

Baromet. 30.2.y Amt of

State of

Weather

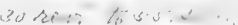

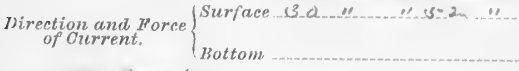

Tille

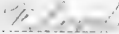

\section{(ieneral Locality}

\section{Position}

Chart usad: Mo

E't'n

Drift: Direction. C Distance

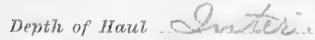

Appurathes and kis used

$1 \leq d$

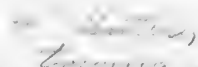

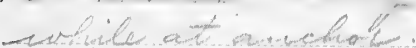

I'ime towins

$h, 10 \ldots$

Remurkss

$-12 \ldots$

-1, 
SOUNDING WIRE.

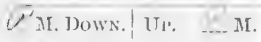

$10400,1,400$
FMS. DREDGIN(: CABLE.

100

200

300

400

500

(i)0

700

$\mathrm{s}(0)$

()(0)

1000

1100)

$1: 200$

1300

1400

1500

1000

$1 \% 00$

1800

1900

2000

2100

2200

$2: 300$

$\because 400$

$\square 500$

2600

$27(40)$

2800

2900

$: 3000$

3100

3200

$: 3: 00$

8100

$330(0)$

$: 3(0)$

3700

33500

:3900

1000

4100

1200

4300

4100

1500

4600

4700

1900

: 1900
M. Dow Un. U.

1:04: $30, \ldots$
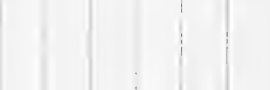


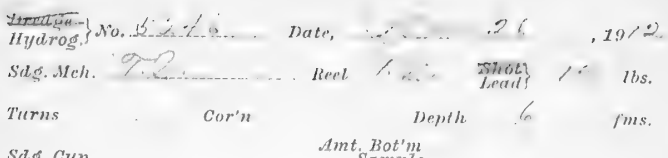

Sdg. Cup

Sample

Character of

Bottom

Thermom, $\left\{\begin{array}{l}\text { Make } \\ \text { No: } 0,0,3\end{array}\right.$

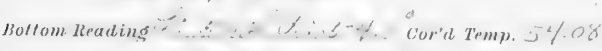

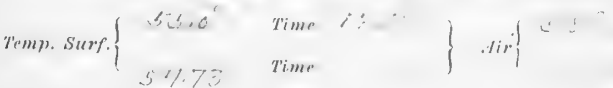

Density: Surf. _..._. . Fims,

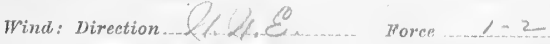

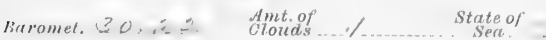

Weather

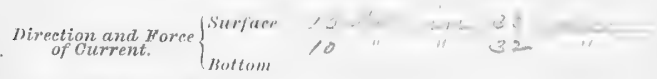

Tille

Gennral Loralily

\section{Josition.}

Chart used: No.

$\pi d l^{\prime} n$

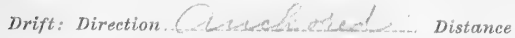

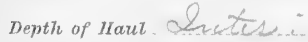

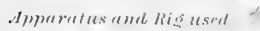

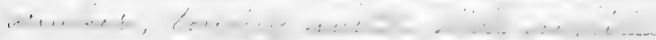

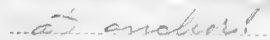

Timetouringi

11. 1 . $m$.

Remirerk:s:

, 


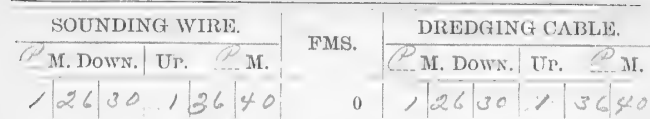

00

200

$\$ 300$

400

is) 0

(i) ()

ז00

$\mathrm{s} 00$

900

1000

110()

1200

1300)

1400

1500

1600

1700

1800

1900)

2000

2100

2200

2300

$\approx 100$

2500

2600

$2 \% 00$

2800

2900)

$: 3000$

3100

3200

$3: 300$

:310)

3500

:B(60)

3ั00

उसOO

:3!not)

1000

.1100

4200

1300

4100

4500

.1600

1700

.1800

(1900) 
$\left.\begin{array}{l}\text { Hundere } \\ \text { Hydrog }\end{array}\right\}$ No. $15: 2,4$

Sig. Mch.

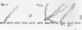

\section{Turns}

Cor')
Date, 2 oze 26 Reet... 2tows. (3) $1 . . "$

fims.

Silf: $C u$

Amt. Bot'm Sample.

\section{Character of}

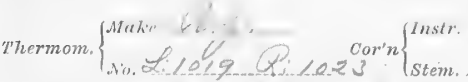

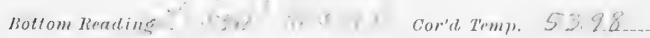

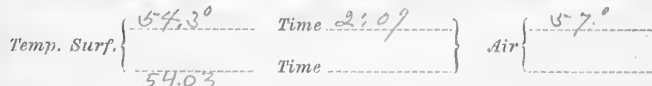
Drusity: surf: fims.

Wind: Direction. 20 Force $1-2$

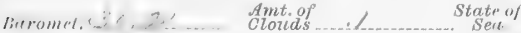

Weather

Direction and Forec
of Current. $\left\{\begin{array}{l}\text { Sufure } \\ \text { Bullom }\end{array}\right.$

rivil $\quad, 0$

Generel Loralily

\section{Position}

(Whort uswel: Ho

Ed'n

Inifl: Hireetion

Distan'e

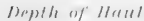

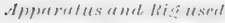

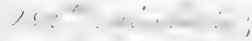

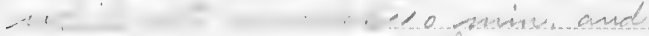

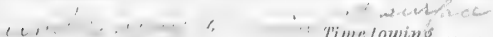

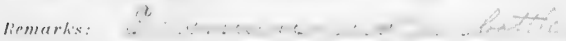
$16-m$

limentis: $\therefore, \ldots, y=0$ 


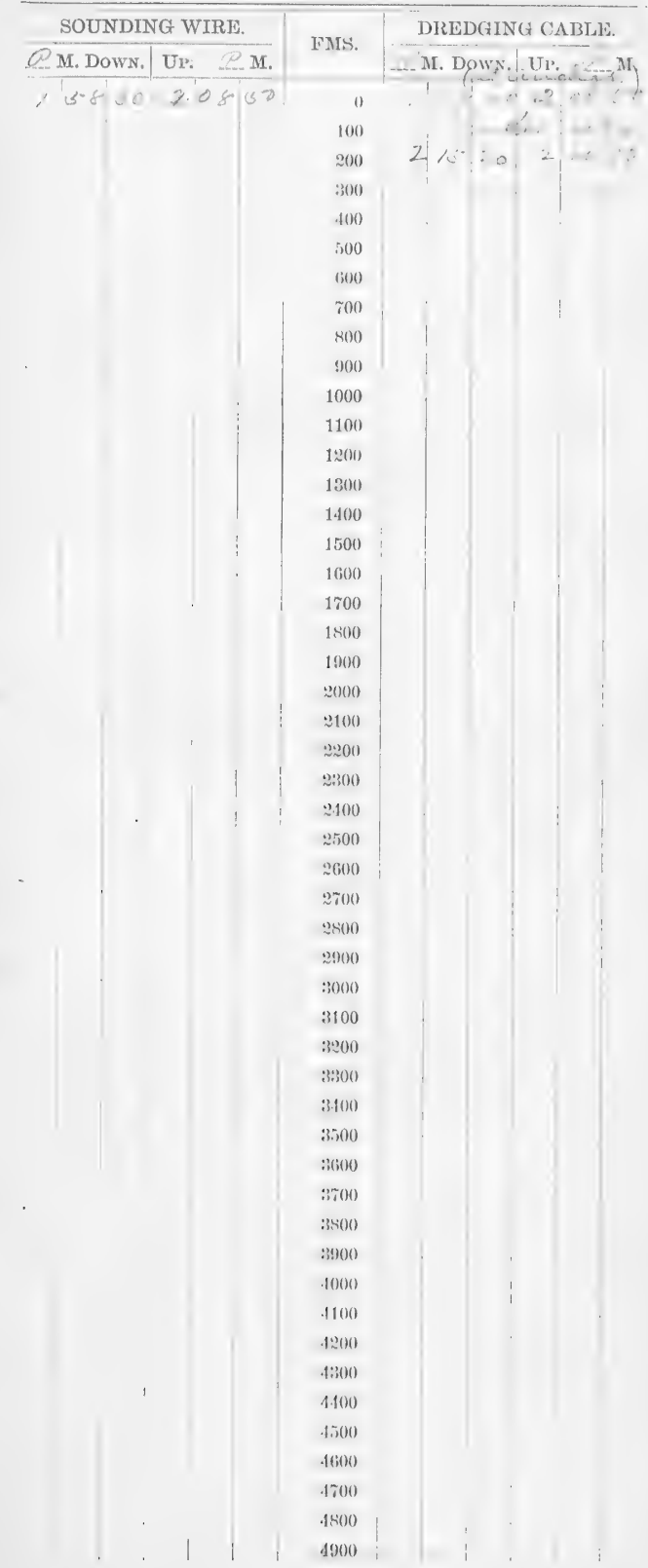




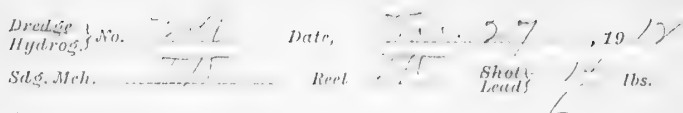

Turns

Cor'n

Depth fins,

Sils. Curp

Amt. Bot'm

Chdracter of

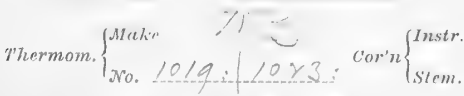

Dollom Readins FS.j.

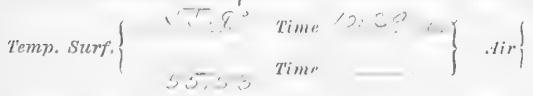

J)hsily: Surf.

fins.

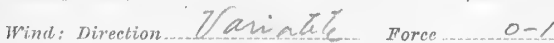

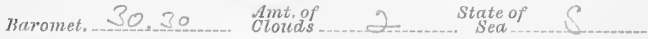

Weather $7 / \mathrm{t} \ldots+$

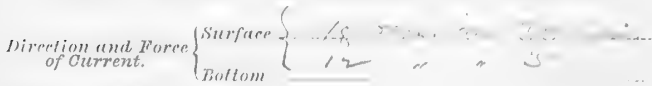
Tide $\quad \ldots \quad \therefore \quad-\square$.

\section{General lorrelity}

Position

Churt usert: : Ais.

It'l'n

l),ill: I)irretiun

Distunte

Denth of Haut

Ap)uralus ant his usral

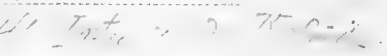

Time towing.. .10.

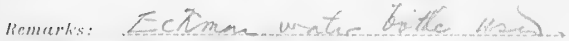
S $, \ldots, i, \ldots$

1

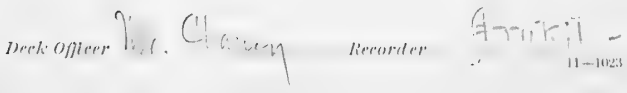


- 11-1023

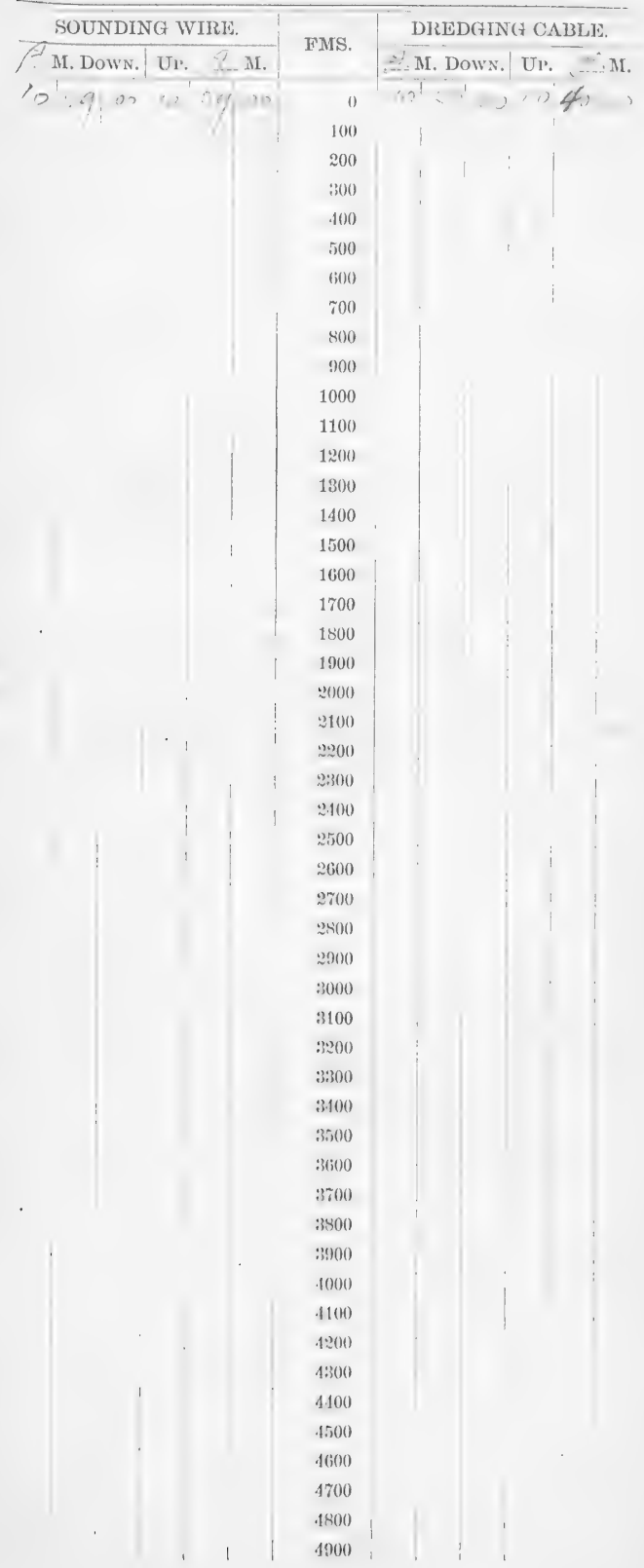




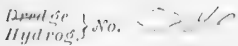

sil E. HrH.

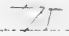

Turns

Cor'n
Date,,$\therefore \ldots+$.

firel , that tis Depth fms.

Sits. Ciup

Amt, $730 t^{\prime} m$.

Character of Bultom

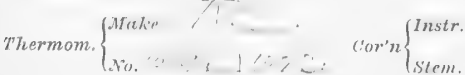

Botlom Rerating

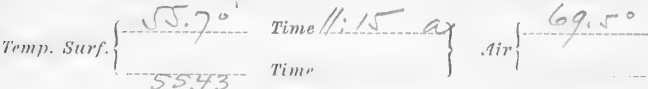
fms.

Density: Surf.

Wind: Direction Dariable porce 0 -

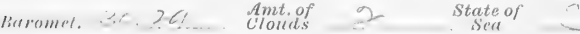

Weather Les

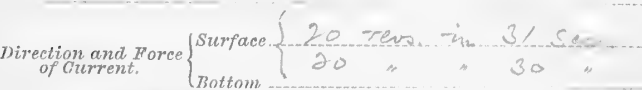

Tide

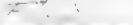

\section{General Locatily}

\section{I'osition}

Chart used: No. I'd'n

Mrifl: Dirretion

Distruen

Denth of Hauz ..... Totes.

Apuratus aud lizizi usert.

Time towing h... $9 . . . m$

liemerkes: :

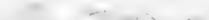

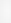


SOUNDING WIRE.

$\rightarrow$ M. DOWN. UP. .I. M. $, 1, \ldots, \ldots,-1,00$

FMś.

DIREDGIN (4 CAISLIS.$$
\text { (1) }
$$

AM. Dows, UP. I. M.

()

100

200

300

400

500

600

\%0)

800

900

1000

1100

$120 \%$

1300

1400

1500

1600

1700

$1 \mathrm{~s} 00$

1900

2000

2100

2200

$2: 300$

2100

2500

2600

2ร(0)

8400

29100

$\therefore(000$

$\$ 100$

:3200

:3300)

3100

3.

$: 3400$

:

3800

$: 3900$

.4000

$\$ 100$

.

4300

4400

4500

(16)0

4700

.4800

4900 


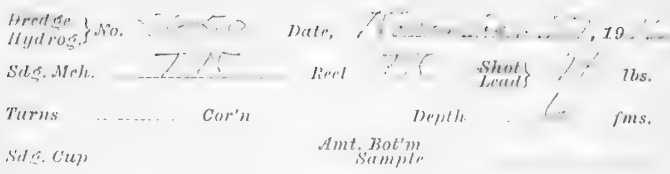

Character of

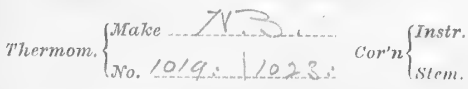

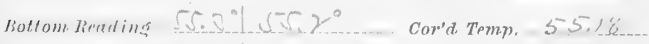

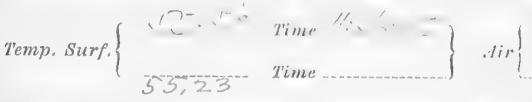

Density: surf.

fms.

Wind: Direction

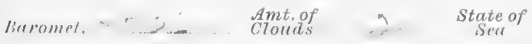

Weather

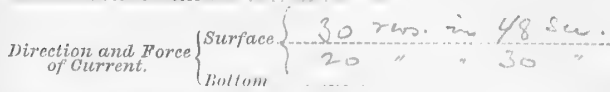
Titer.

General Locality

Position

Chart used: No. $E d^{\prime} n$

Drift: Direction Distance

Depth of Haul

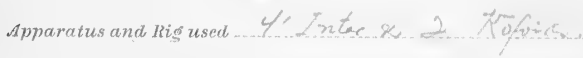

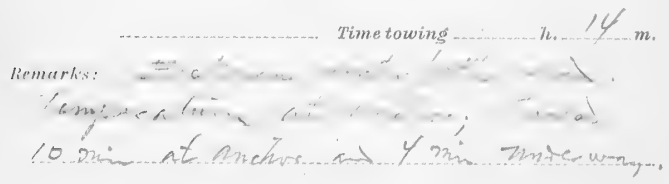

Derek offerer ?', '. ....

liecortes.

iii 1028 
$11-1023$

SOUNDING WIRE.

\#M. Down. UY. ... M.

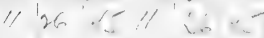

FNS. DIRIDGING CAIBLE.

100

200

30()

$\$ 00$

500

(i) 00

$\% 00$

800

900

1000

1100

12()

1300

1400

1500

1600

1700

1800

1900

2000

2100

2) 0 이

$2: 300$

2100

250

2600

2ริt)

2800

ำ()

$30(00)$

3100

$\$ 3000$

:3i300)

3100

Bijo0

: $3(i 0) 0$

i霍()

: 3800

:3900

.1000)

$.1100)$

1:)(1)

4:30)

4100

1in $(1)$

460

4700

4800

1900

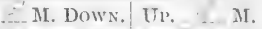

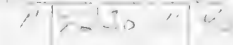

1 1

;
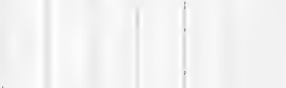


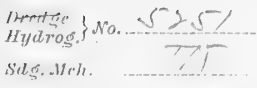

Sill $. .11+h$.

$C \omega r^{\prime} n$

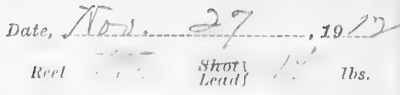

nepth 6 .

fims.

$$
\text { . Int limtin, }
$$

Silss. (iu)

Character of Bollom

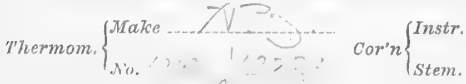

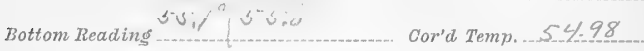

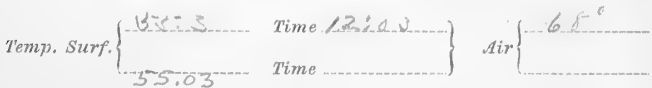

Density: Surf.

fins.

Wind: Direction Cos Force 0

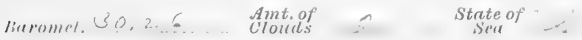

\section{Weather}

Direction and Force
of Durrent. $\left\{\begin{array}{l}\text { Surface } \\ \text { Bottom }\end{array}\right.$ Tilie

\section{Gumpal Loralify}

\section{Position}

Chrrt used: No.

lid'n

Hrift: Diverlion

Dis/anum

Depth of Haut... T2z

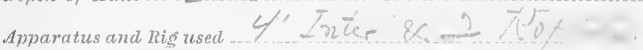
$72 ., 2,2, m$.

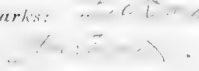




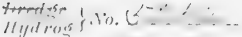

J)ate, . . : ...

$\because \% \quad, 10=$

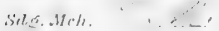

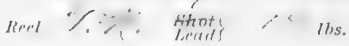

Turns

Cor'n

Depith

fins,

Sclg. Cup

Anut. Bot'm
Sample

Character of

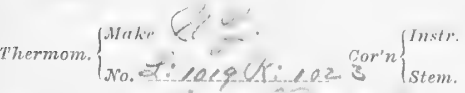

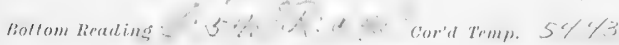

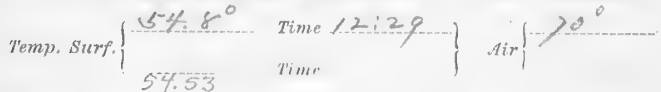

linsity: sitr/:

fills.

Wind: Direction.. (Yothree Force.....

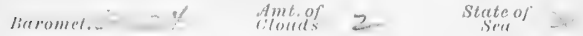

Weather ...

Direction and Force $\left\{\begin{array}{llll}\text { Surface } 20 & 0 & \text {, } 30\end{array}\right.$ of Current.

Hotterm

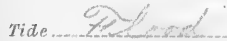

(innerel Loralill!

\section{Position}

Chart userl: No $H d^{\prime} n$

Drift: Direction

Distance

Inepith of Haul

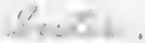

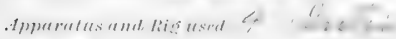

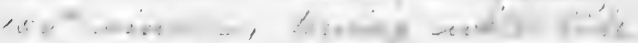

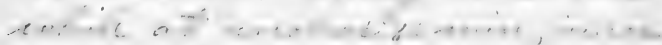
- . , m,

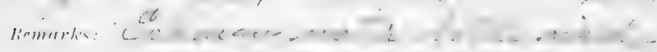


SOUNDING WIJE:

Ch. DOWN.| U1. $12.18|00: 12| 2810$
Fม⿱

1)REDGING ('AIBTA:

7... M. Dow. Ur. M.

$0|/ 2, / 4| 00 / 2 \mid 3500$

(11)

s00

:001)

$.106)$

500

(i)

ร00

si( )

900

1000

110 ()

12(1)

1300)

1400

1500

1600

1700

1800

म!900

$\because 000$

2100

2ख00

2:300)

$\because 100$

2500

$\therefore(60)$

$20(4)$

$\because 400$

모의

:(0)(*)

$\$ 3100$

:3(1)

(3300

:1900

:3(3)

:(1)0

:30)

:3400

:?(10)

$.10(4)$

.8100

1901

1300

1. 100

$1500)$

(1):

4.

.1800

.9000 


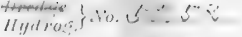

Silf... Heth.

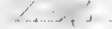

Cor'n jut八,

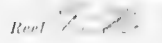

$\therefore \%$ $1 !) \div$

Turns

$$
\text { Depth }
$$

Sheol lexudf

11 is.

Sils. (iu)

Amt. Irot'm

Character of Bottom.

Thermom. $\left\{\begin{array}{l}\text { Make } \\ \text { No.a. }\end{array}\right.$ Cor'n $\begin{array}{l}\text { Instr. } \\ \text { stem. }\end{array}$

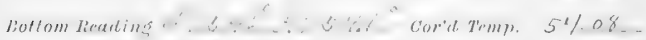

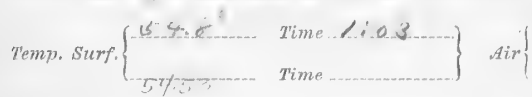

Insily: sin\%: jims.

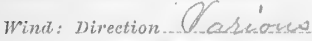
Force $0-1$

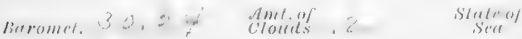

Hetather

$$
18-3 .+i \quad 11+2
$$

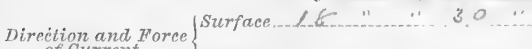
of Current. Bioffom

Tiale of

General bosatity

\section{Josition}

Chavt used: No. $x d ' n$

Drift: Direction

Distance

Depth of Haut elect.

spmaratus and lition IIsmet

$1: \therefore$ - $\therefore:$ :

?.

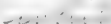

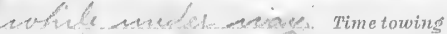
7. $13 . m$. linmertis: $c^{6}$

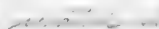
fins. 

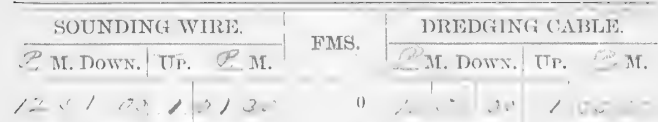

()

100

$200)$

:3(H)

.1130

500

li)( )

\%()

S()0)

()0()

1000

1100

1200

1300

1400

1500

1600

1700

1800

1900

2000

2100

2200

2300

2400

2500

2000

2700

2800

2900

3000

8100

3200

3300

3400

3500

3600

3700

3800

33900

4000

4100

.4200

4300

4400

4500

1600

4700

4800

4900 


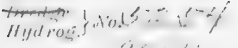

Cor'n
Dite, $<\because \cdots, \ldots ; 0,10<2$

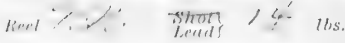

I)

$\therefore$ Si. C'ul

Ant Bot'm

('hariscleref

lioltom

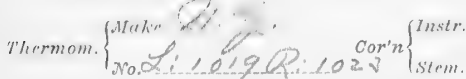

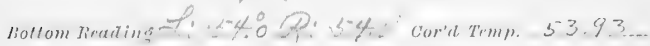

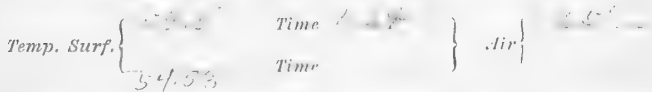

l)ensityf: sunf.

fins.

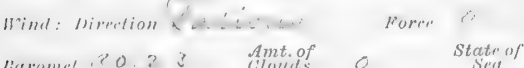

Ifexther - $\therefore \therefore$

,

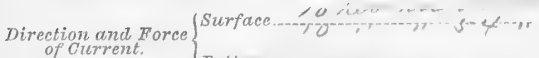
of Current. $\quad$ Bullum

Tide

$$
\text { Boliom }
$$

tr... $\because \because \ldots+2 \div \div$

\section{Generul Lorilill}

Posiliom

that usert: tho.

I:I I

Drift: Direction

Distance

Depth of Haut Q2-ares.

Alpunatus and lititused

$, 1, \ldots+\ldots$.

$, \ldots, \ldots, \ldots, \ldots, \ldots$

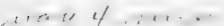

Time forvins

h. $; m$

Remartes:

$$
i^{7}
$$

. $, 6, j$ 
SOUNDDNG WIRli.

$\therefore$ MI. DOWN.|Th. \&.. M. $1 \therefore 1,2320$
DREDGING ( $A B L F$

M. Dows. UI. - II.

()

100

200

:3(0)

.100

500

1i()( )

70)(

Sil)

(1)()

1000

1100

12(i)

130()

1,100

1500

1600

1700

1800

1900

2001

2100

2000

$2: 301)$

3100

30()

: $8(1)$

$2 \pi 00$

2800

?)(30)

$\because 000$

3100

:3) $(x)$

:is: $)(0$

3900

:3.700

:3600

:ivit)

:ival)

$: 3 !)(1)$

I(1)(1)

$110(1)$

12)( $)$

$1: 300$

4.100

.1500

itivo)

$17(1)$

1S(1)

. 1000
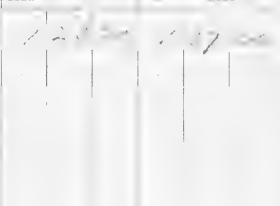


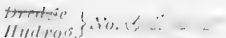

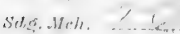

Turnsi

$C\left(r r^{\prime \prime \prime}\right.$

I) 10

\section{Sid (s. $(u)$}

Character of Bintiom

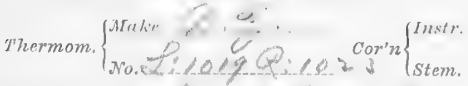

Lotfom Readins . \$1 :

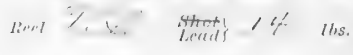

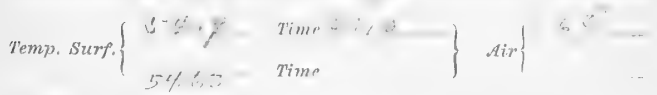

l)ensily: Surf:

firs.

Wind: Direction. Force 0.1

Baromet. 30.22 Amt.of 2 State of

Wrather..?

Direction and Force \{surfrem of Current. liottum

Tille

\section{General Locality}

\section{l'osition}

Ghert usert: in

lith

Hrifl: Jivetimn

Depth of Haul. OQntes.

Jisturier

Ap)

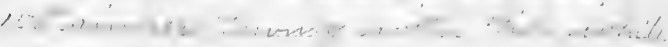

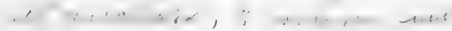

Time towing

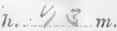

liemartis:

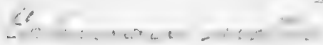

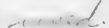




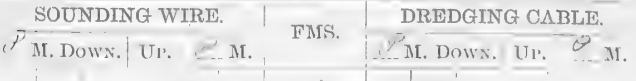

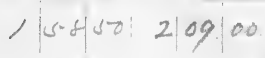

200

:300)

.100

500

(ii)()

700

8())

(90)

1000

1100

12(1)

1300

1400

1500

1000

1700

1800

1900

$: 000$

2100

2000

$23(1)$

$2100)$

2500

20

27(19)

2800

эอติ

$: 300$

3100

:30)(

: $: 3300$

$\because 100)$

8300

\#is00

:1700)

:300)

:390र

.10000

.1100

4:00)

1:300)

1900

.1500

1600

.1700

4800

4900 
7) 7 - $-\cdots$

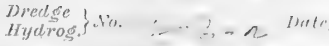

27,10 ;-

Sile. Hoth.

lin,

Strot? lis lls.

Turns

Cor'n

Depth

fins.

Silta (is)

Amt. Bot'm

Character of

Whermom. $\left\{\begin{array}{l}\text { Make ..... } \\ \text { No, }\end{array}\right.$

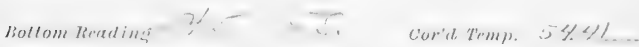

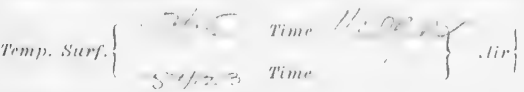

In'msit!: : ster\%:

fims.

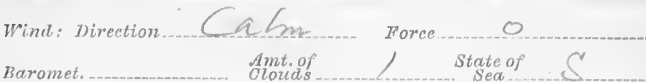

W"rather

Direction and Iforce
of Current. $\left\{\begin{array}{l}\text { Suritas } \\ \text { Botfom }\end{array}\right.$

Tilie

\section{General Loratity}

Position

UWart usert: So

Ed'n

Drift: Direction...... Dnetsons.

Depth of IIaul

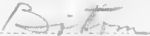

Distinnce

Apparatus and litis used

Time towing

$m$.

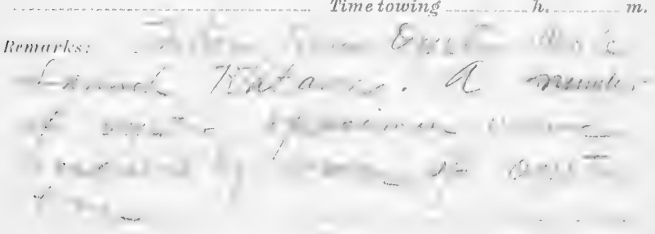

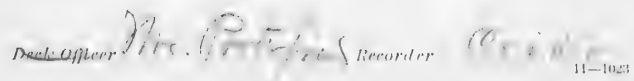


SOINNIDNA IVIRT.

Cl, MT Howx. UH. CLM.

101911109
F.IS,

DREDCFING (. IILA

M. InIS. I'P. M.

100

290

:390)

100

500

(50)

ז00

s(9)

(11)0

1000

1100

12001

1300

1400

1500

1600

1700

1800

190019

2000

2100

2200

$2: 00)$

$\because 100$

500

2000

2700

2800

2900

$: 000$

$\$ 100$

$: 300$

:3isol

3100

3500

3600

$3 \% 00$

$\because 500$

:890)

.1000

$\$ 100$

400

4:800

1100

4500

f(t)

1\%(1)

4500

(1900) 


$$
\mathrm{H} .5256 \mathrm{~b}
$$

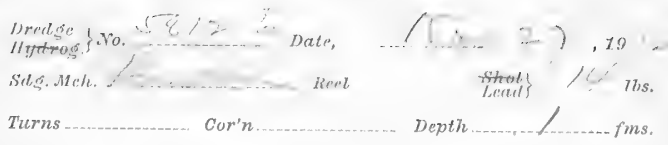

Sals. Cup Amt. Bot'm

Character of Bottom

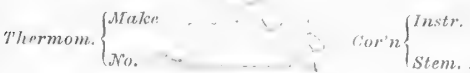

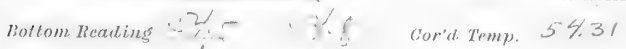

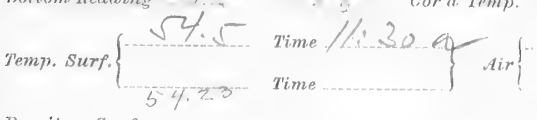

Density: Surf.

fins.

Wind: Direction

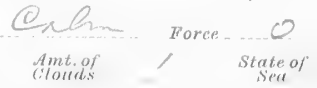

Buromet.

Heather

Direction and Force
of Current. $\left\{\begin{array}{l}\text { Surface } \\ \text { Bottom. }\end{array}\right.$

Tide

General Localily

Position

Chart used: No.

$E d$ 川

Dnift: Direction

Depth of Maul

Apparatus and Rid used

liemurks: 
SOUNDING WIRE.

M. Down, Jp。 M. $1120|\ldots| 1 / 30$
T)IEDGING C.IBLE.

M. I)แ⿴囗十 I'P. II. 0

100

20()

:3(i)

10)

5)(1)

(iti)

(i)()

Si(i)

9)(1)

$10(1)$

1100

$1 \div(1)$

19:0)

1.100

1500

16:00

$1 \% 00$

1

1900

2000

2100

요이

:3:00

$\because 110)$

:3010

$\because(6) 0)$

$2 \%(100$

2800

2900

3000

3100

$: \cdots 00$

:3:300)

$:: 100$

3300

$: 3600$

$:$ :

अथ0

$: 900$

1000

.1100

19(1)

1::01)

4100

1500

A(tio)

$4 \% 00$

4800

4900 


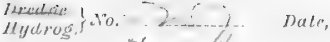

Sdg. Mch.

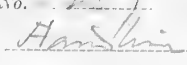

Reel

Stoots, $;$ ilis.
Turns
Nepth
fins.
Sil,., Cup Amt, Bot'm
Character of buttom
h.sem

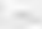
Thermom. $\left\{\begin{array}{l}\text { Hake: } \\ \text { Noo. }\end{array}\right.$
Cor'n $\left\{\begin{array}{l}\text { Instr. } \\ \text { stem. }\end{array}\right.$

Jottom Reading Cor'd Temp.

Temp. Surf.

Time

Time

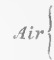

Density: Surf.

Wind: Direction

fins.

Baromet.

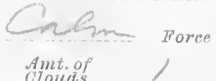

Heather

State of

,

Direction and Force
of Current. $\left\{\begin{array}{l}\text { Surficr. } \\ \text { Dottom }\end{array}\right.$

Tide

General Locality

Josition

Chart used: No. $E d l^{\prime} n$

Drift: Direction

Distance

Depith of Haut

dpparalus aud Ris usrd
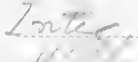
S()lNI)IN(* WTRI:

.... M. Down. \{\}$_{1}$. ... Ml.

IMIS.

()

1(1)

200

$?(0)$

.100

inot)

(i)ic)

io()

sino

())( )

$10(1)()$

$111)$

(이)(1)

(i⿱⺈)(1)

1400

1500

1(it)

$1 \%(1)$

18(0)

$18(1) 0$

2000

:100

$2: 200$

s:300

$\because 100$

$\because 500$

2600

3200

저아

2000

:300)

3100

:3:00

:3:300

:1101)

:3500

:3600

:300

3800

:3:100

4000

$\$ 100$

.9200

1:300

.1100

.1500

4600

4700

.1800

(900)
DREDCHNG CABLAL.

QM-Down. UP. Gune.

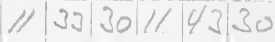

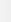




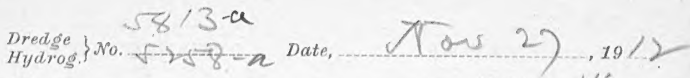
Sd. Meh. Reel Shoot 1/ 1 to s.
Leads Turns Corn Depth fms.

Sag. Cup Amt. Bot'm
Sample Character of
Bottom

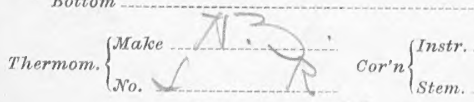

Bottom Reading $\sqrt{5}, 2 \sqrt{3}, 2$ Cord Temp. 5476 Temp. Surf. $\left\{\begin{array}{l}\sqrt{5}, 2 \text { Time } 12: 58 / \\ 54.93\end{array}\right\}$ Time Air Density: Surf. fms, Wind: Direction Force $\quad 0-1$ Baronet. Amt. of State of
Sea Weather Direction and Force
of Current. $\left\{\begin{array}{l}\text { Surface } \\ \text { Bottom }\end{array}\right.$ Tide General Locality

Position

Chart used: No. $E d ' n$

Drift: Direction Distance

Depth of Haul 7 ar ti

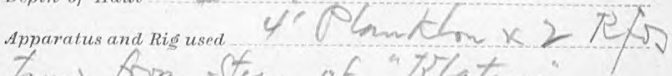

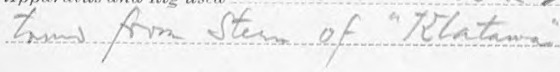

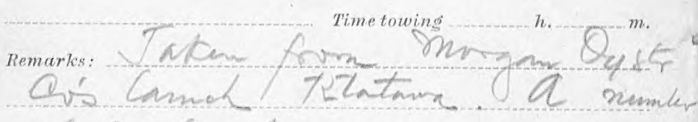
of oyster fpecmo unesco

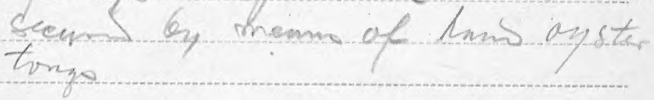

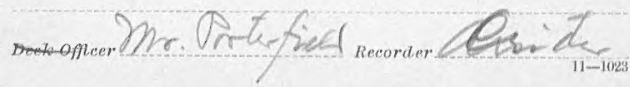


FMS.

Q M. Down. UR. QM. is 4800 is 18 ao

0
DREDGING CABLE.

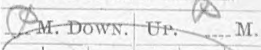

100

200

300

400

500

600

700

800

900

1000

1100

1200

1800

1400

1500

1600

1700

1800

1900

2000

2100

2200

2300

2400

2500

2600

2700

2800

2900

3000

8100

8200

3800

3400

3500

3600

3700

3800

3900

4000

4100

4200

4300

4400

4500

4600

4700

4800

4900

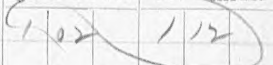

I. 


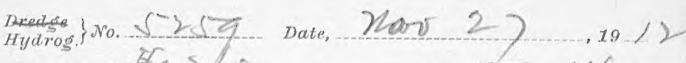

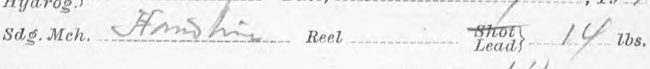
Turns Corn Depth L fms.

Sd. Cup Amt. Bot'm

Character of
Bottom

Thermom. $\left\{\begin{array}{l}\text { Make ... Corn }\left\{\begin{array}{l}\text { Instr. } \\ \text { So. }\end{array} \text { Stem. }\right.\end{array}\right.$

Bottom Reading Cor'd Temp.

Temp. Surf. $\left\{\begin{array}{cc}56.0 & \text { Time } \\ 55.73 & \text { Time }\end{array}\right.$

Density: Surf. fms.

Wind: Direction.

Baronet. Amt. of Force Q

Weather

Direction and Force
of Current, $\left\{\begin{array}{l}\text { Surface } \\ \text { Bottom. }\end{array}\right.$

ride

General Locality

Position

Chart used: No. Eden

Drift: Direction

Depth of Haul Inter.

Distance

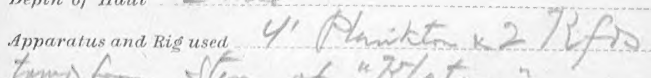

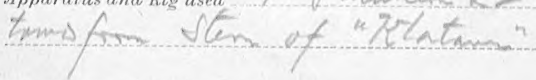

Time towing

n. $\bigcirc$.

Remarks :

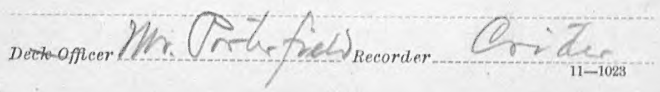


SOUNDING WIRE.

M. Down. UP. _... M.
FMS. DREDGING CABLE.

G. DoWn. UP. OM.

01 or or 1,1200

100

200

300

400

500

600

700

800

900

1000

1100

1200

1800

1400

1500

1600

1700

1800

1900

2000

2100

2200

2300

2400

2500

2600

2700

2800

2900

8000

3100

8200

3800

8400

3500

3600

8700

3800

3900

4000

4100

4200

4300

4400

4500

4600

4700

4800

4900 